\title{
Pain management in the neonatal piglet during routine management procedures. Part 1: a systematic review of randomized and non-randomized intervention studies
}

\author{
R. S. Dzikamunhenga ${ }^{1}$, R. Anthony ${ }^{2}$, J. Coetzee ${ }^{1}$, S. Gould ${ }^{1}$, A. Johnson ${ }^{1}$, \\ L. Karriker ${ }^{3}$, J. McKean ${ }^{1 \dagger}$, S.T. Millman ${ }^{1,4}$, S. R. Niekamp ${ }^{5}$ and A. M. O'Connor ${ }^{1 *}$ \\ ${ }^{1}$ Department of Veterinary Diagnostic and Production Animal Medicine, College of Veterinary \\ Medicine, lowa State University, Ames, lowa, USA \\ ${ }^{2}$ Philosophy Department, University of Alaska Anchorage, Alaska, USA \\ ${ }^{3}$ Department of Veterinary Diagnostic and Production Animal Medicine, Swine Medicine Education \\ Center, College of Veterinary Medicine, lowa State University, Ames, lowa, USA \\ ${ }^{4}$ Department of Biomedical Science, Department of Veterinary Diagnostic and Production Animal \\ Medicine, College of Veterinary Medicine, lowa State University, Ames, lowa, USA and \\ ${ }^{5}$ National Pork Board, Des Moines, lowa, USA
}

\section{Received 9 February 2014; Accepted 30 April 2014}

\begin{abstract}
Routine procedures carried out on piglets (i.e. castration, tail docking, teeth clipping, and ear notching) are considered painful. Unfortunately the efficacy of current pain mitigation modalities is poorly understood. The aim of this systematic review was to synthesize the existing primary scientific literature regarding the effectiveness of pain management interventions used for routine procedures on piglets. The review question was, 'In piglets under twenty-eight days old, undergoing castration, tail docking, teeth clipping, and/or methods of identification that involve cutting of the ear tissue, what is the effect of pain mitigation compared with no pain mitigation on behavioral and non-behavioral outcomes that indicate procedural pain and post-procedural pain?' A review protocol was designed a priori. Data sources used were Agricola (EBSCO), CAB Abstracts (Thomson Reuters), PubMed, Web of Science (Thomson Reuters), BIOSIS Previews (Thomson Reuters), and ProQuest Dissertations \& Theses Full Text. No restrictions on year of publication or language were placed on the search. Eligible studies assessed an intervention designed to mitigate the pain of the procedures of interest and included a comparison group that did not receive an intervention. Eligible nonEnglish studies were translated using a translation service. Two reviewers independently screened titles and abstracts for relevance using pre-defined questions. Data were extracted from relevant articles onto pre-defined forms. From the 2203 retrieved citations forty publications, containing 52 studies met the eligibility criteria. In 40 studies, piglets underwent castration only. In seven studies, piglets underwent tail docking only. In one study, piglets underwent teeth clipping only, and in one study piglets underwent ear notching only. Three studies used multiple procedures. Thirty-two trial arms assessed general anesthesia protocols, 30 trial arms assessed local anesthetic protocols, and 28 trial arms assessed non-steroidal antiinflammatory drugs (NSAIDs) protocols. Forty-one trial arms were controls where piglets received either placebo or no treatment. Forty-five outcomes were extracted from the studies, however only the results from studies that assessed cortisol (six studies), $\beta$-endorphins (one study), vocalisations (nine studies), and pain-related behaviors (nine studies) are reported.
\end{abstract}

*Corresponding author. E-mail: oconnor@iastate.edu

$\dagger$ Deceased: 10 April 2014

The online version of this article is published within an Open Access environment subject to the conditions of the Creative Commons Attribution licence http://creativecommons.org/licenses/by/3.0/ 
Other outcomes were reported in only one or two studies. Confident decision making will likely be difficult based on this body of work because lack of comprehensive reporting precludes calculation of the magnitude of pain mitigation for most outcomes.

Keywords: castration, tail docking, pain, anesthesia systematic review, meta-analysis, swine

\section{Introduction}

In swine production, piglets undergo castration, tail docking, teeth clipping, and identification with ear notching or ear tagging. These procedures are considered painful. Although available and increasingly warranted by the public in other countries, pain mitigation strategies during these procedures are not routinely provided to piglets in the United States (USA). As the US public becomes more cognizant about on-farm practices, they will likely want to see more humane practices encouraged and enforced, if recent ballot initiates and state legislations for better animal welfare are any indication of future trends. However, the current absence of provision may be explained by historical precedent, economic barriers, impracticality, uncertainty about need, uncertainty about the legality of drug usage, and uncertainty about the efficacy of pain mitigation strategies. To address the uncertainty about efficacy, we conducted a systematic review, reported here, of the available scientific evidence regarding the impact of interventions on pain in piglets undergoing castration, tail docking, teeth clipping, and identification with ear notching. We sought to report summary measures of efficacy and to identify research gaps where appropriate. The systematic review formed part of the knowledge base used for the development of recommendations about the use of pain mitigation in piglets in the USA in a project funded by the National Pork Board. Recommendation development is a multistep process described by Oxman et al. (2006).

The specific review question was, 'In piglets that undergo castration, tail docking, teeth clipping, and/or methods of identification that involve cutting of the ear tissue, such as ear tagging and ear notching, what is the effect of pain mitigation (e.g. general anesthesia and local anesthesia (NSAIDs)), compared with no pain mitigation, on behavioral (e.g. postures and vocalisations), and non-behavioral (e.g. blood cortisol, norepinephrine, $\beta$-endorphin levels) indicators of procedural pain, assessed within $60 \mathrm{~min}$ of the procedure, and postprocedural pain, assessed between 1 and $24 \mathrm{~h}$ of performing the procedure?'.

\section{Materials and methods}

\section{Protocols and registration}

The development of the review protocol was consistent with previously published guidelines (EFSA, 2010;
Higgins and Green, 2011). The review protocol was developed using input from the authors and critiqued by external participants. Minor modifications were made based on the external participant comments; the protocol was considered finalized on 13 December 2012.

Post-finalization of the protocol, some changes were made: (1) to extract data from figures, (2) to have an external expert determine post hoc which of the extracted behavior state and event data were 'pain-related' behaviors, and (3) to extract data if studies described blinding and blocking or stratification to limit confounding in addition to randomization. The reason for the first change was the large amount of data that would have been excluded if data in figures were excluded. For the second change, there was a large variation in terminology used to describe behavioral outcomes, which was only apparent after data extraction. For example, some authors consolidated pain behavioral outcomes and reported them as index scores, other parameters reported were not necessarily identified as relevant pain responses. When multiple relevant pain behaviors were provided, we randomly selected one behavioral outcome within painrelated behaviors associated with avoidance or removal of noxious stimuli. For example, although we recognized that pain can result in general changes in time budgets, we assumed behavior responses directed to the rear quarters (tail flinching, tail wagging, tail rubbing, kicking, scooting, and easing the quarters) would be more sensitive measures of pain resulting from castration and tail-docking surgeries, versus behaviors associated with exploratory or feeding motivational systems. The third change was necessitated by the obvious omission of important design characteristics by some studies. The changes are reflected in this paper.

\section{Eligibility criteria for studies}

The review question was defined in terms of a PICOS format: the population (P), intervention (I), comparator (C), outcome (O), and study design (S) (Bekelman and Yahalom, 2009).

\section{Population}

The study population was defined by two components: (1) the animals and (2) the procedures of interest. Relevant study animals were limited to piglets under the age of 28 days. Piglets described as neonatal, lightweight, suckling, or pre-weaned were also considered part of the 
Table 1. List of outcomes extracted as required by the protocol

\begin{tabular}{|c|c|}
\hline Behavioral outcomes & Non-behavioral/physiological outcomes \\
\hline $\begin{array}{l}\text { Vocalisation - call duration } \\
\text { Vocalisation - call rate } \\
\text { Vocalisation - main frequency } \\
\text { Vocalisation - peak amplitude } \\
\text { Vocalisation - peak frequency } \\
\text { Activity event - defecation } \\
\text { Activity event - escape attempts } \\
\text { Activity event - urination } \\
\text { Activity event - other } \\
\text { Activity state - lying } \\
\text { Activity state - playing } \\
\text { Activity state - running } \\
\text { Activity state - sitting } \\
\text { Aggression event } \\
\text { Avoidance event } \\
\text { Body movement event - ear flicking } \\
\text { Body movement event - head shaking } \\
\text { Body movement event - rear end movement } \\
\text { Body movement event - other } \\
\text { Feeding event - suckling/nursing } \\
\text { Feeding event - teat seeking/udder mouthing } \\
\text { Feeding event - teeth champing/chewing } \\
\text { Feeding state - suckling/nursing } \\
\text { Feeding state - teat seeking/udder mouthing }\end{array}$ & $\begin{array}{l}\beta \text {-endorphins }{ }^{1} \\
\text { Body temperature } \text { Cortisol }^{1} \\
\text { Electrocardiography (ECG) } \text { Electroencephalography (EEG) } \\
\text { Heart rate } \\
\text { Respiratory rate } \\
\text { Norepinephrine }^{1} \\
\text { Adrenocorticotrophic hormone (ACTH) } \\
\text { Epinephrine } \\
\text { Haptoglobin } \\
\text { Substance } P\end{array}$ \\
\hline
\end{tabular}

Reported within 60 min of the procedure and/or between 1 and $24 \mathrm{~h}$ of the procedure.

${ }^{1}$ Final outcomes assessed provided there was sufficient data.

relevant population. Miniature pigs, pot-bellied pigs, and teacup pigs, regardless of age, were excluded. No other restrictions were placed on the study animals (i.e. country, year, production system, or management environment). The procedures of interest were castration, tail docking, teeth clipping, and any method of identification that involved cutting of the ear tissue (i.e. ear tagging and ear notching). Microchip insertion, tattooing, and iron injection were not within the scope of this review. Where piglets received single or multiple relevant procedures, the study was included, provided piglets in the control and treatment groups received identical procedures.

\section{Intervention(s)}

Any intervention that aimed to mitigate pain measured within $60 \mathrm{~min}$ of the procedure or between 1 and $24 \mathrm{~h}$ post-procedure was considered relevant.

\section{Comparison}

Comparison groups of interest were similar animals undergoing the same procedure with no intervention. We did not exclude studies based on the type of control used. For example, consider a study with two arms where one group of animals was castrated only, with no intervention, and the other group received a pharmacological intervention $30 \mathrm{~min}$ prior to castration. Despite the differences in handling of animals between the groups, the castrated-only group was considered a relevant control group. Results reported in sham arms (i.e. a negative control, in which animals were handled as if the procedure was conducted, when it was not), were not relevant to the review.

\section{Outcome(s)}

Outcomes of interest were those that assessed procedural pain (within $60 \mathrm{~min}$ of the procedure) or post-procedural pain (from $1 \mathrm{~h}$ to $24 \mathrm{~h}$ after the procedure). For descriptive purposes, outcomes were categorized as behavioral or non-behavioral (Table 1). Behavioral outcomes were classified as either states or events as described by Martin and Bateson (2007). Adverse-event outcomes for which data were extracted included herniations, mortalities, morbidities, and problems with wound healing. Production outcomes were not included.

\section{Studies}

Parallel or non-parallel, randomized or nonrandomized, and controlled trials with at least two arms were relevant study designs. Potential sources of data were published studies, reports, dissertations, and conference proceedings that were at least 475 words because short proceedings often lack comprehensive reporting (Brace et al., 2010). No restrictions on year of publication or language were placed. Relevant non-English language publications 
Table 2. Search strategy for CAB Abstracts (Thomson Reuters) on 12 October 2012

\begin{tabular}{lll}
\hline Line & Search terms & $\begin{array}{l}\text { Number of } \\
\text { citations }\end{array}$ \\
\hline 1 & $\begin{array}{l}\text { pigs OR pig OR swine OR hogs OR hog OR piglets OR piglet (Topic search) } \\
\text { pain OR stress* OR well-being OR welfare OR anesthesia OR anesthesia OR anesthetic OR }\end{array}$ & 267,866 \\
2 & $\begin{array}{l}\text { analgesia OR analgesic (Topic search) } \\
\text { tail docking OR tail resection OR docking OR castration OR castrating OR castrated OR } \\
\text { orchiectomy OR teeth clipping OR tooth resection OR teeth resection OR tooth clipping OR } \\
\text { tooth OR teeth OR tooth grinding OR clipping OR ear notching OR notching OR ear tagging } \\
\text { (Topic search) } \\
\text { 1 and } 2 \text { and 3 }\end{array}$ & 54,340 \\
\end{tabular}

were translated using a certified language translation service (http://www.languagescientific.com/).

\section{Information sources}

Six electronic databases were searched on 12 October 2012, and updated on 13 December 2012: CAB Abstracts (Thomson Reuters, 1910-2012), BIOSIS Previews (Thomson Reuters, 1926-2012), Web of Science (Thomson Reuters, 1900-2012), PubMed (1940-2012), Agricola (EBSCO, 1970-2012), and ProQuest Dissertations \& Theses Full Text (1743-2012). Conferences, symposia, and workshop proceedings were available in the Swine Information Library through the American Association of Swine Veterinarians website (http://www.aasv.org/ library/swineinfo/). Some proceedings were indexed in CAB Abstracts, which were expected to be captured by the search strategy described below. The following journals and proceedings from conferences, workshops, and symposia were considered: Journal of Swine Health and Production (JSHAP), American Association of Swine Veterinarians (AASV) Conference proceedings, International Pig Veterinary Society (IPVS) Conference proceedings, Allen D. Leman Swine Conference, International Society for Applied Ethology, International Conference (formerly Workshop) on the Assessment of Animal Welfare at Farm and Group Level: (WAFL), and the Universities Federation for Animal Welfare. Where possible, we also searched the reference lists of relevant reviews and publications that were identified.

\section{Search}

The search strategy was designed to identify: (1) piglets under 28 days of age, (2) pharmacological or nonpharmacological pain mitigation strategies, and (3) the procedure applied. The CAB Abstracts search strategy is shown in Table 2. The search string was applied to search PubMed, Agricola, Web of Science, BIOSIS Previews, and ProQuest Dissertations \& Theses Full Text. Electronic citations obtained were stored in Endnote $\mathrm{X} 6{ }^{\circledR}$
(Thomson Reuters ${ }^{\circledR}$, 2012). Duplicates were removed in EndNote ${ }^{\mathbb{R}}$ and DistillerSR ${ }^{\mathbb{R}}$ (Evidence Partners, Canada).

\section{Study selection}

Assessment of the screening questions involved independently testing them on 20 randomly selected abstracts by the review team to assess inter-rater reliability. The questions were modified until inter-rater reliability was $100 \%$ for 20 abstracts (i.e. $\kappa=1.0$ ). All citations were then independently assessed for relevance by two reviewers using the titles and abstracts (where available) only. The screening questions were:

1. Does the citation describe a primary research study where the study population is pigs less than 28 days of age or is described as piglets, suckling, pre-weaned, or of a weight consistent with those populations?

2. Does the citation describe a primary research study where the study population is subjected to castration, tail docking, teeth resection, ear notching, and/or ear tagging?

3. Does the citation describe a primary research study where the aim is to assess any intervention designed to mitigate the pain associated with the procedure?

4. Does the study describe a group of pigs undergoing the same procedure(s) but not receiving the intervention?

5. Does the study assess outcomes that measure the pain experience during the procedure or within $24 \mathrm{~h}$ of the procedure?

When both reviewers responded 'No' to at least one of the above questions, the citation was excluded from the review. The response that triggered exclusion is not reported. Conflicts between reviewers were resolved through discussion. Where it was not possible to answer the questions based on the abstract or title, the full article was screened. Translations of non-English publications that appeared relevant were performed by Language Scientific Inc. Medford, MA and certified as accurate through a statement released on 27 December 2012. 


\section{Data collection process}

Data extraction forms were piloted on several articles. Two members of the review team extracted data independently from eligible studies using standardized forms in DistillerSR ${ }^{\circledR}$, available at https://www.systematicreview.ca. If more than one source described the same study, information from both sources was used to obtain the most complete description of the study. After data extraction, one reviewer identified disagreements and reevaluated the article, then if the source of disagreement was not a simple transcription error or similar, the paper was discussed with the other reviewer to resolve the conflict. No authors were contacted to obtain missing data or clarify existing data. After conflict resolution, the completed data extraction form and publication were sent to the co-authors and external experts involved in other aspects of the larger project. Each expert was assigned up to three studies to verify the accuracy of the extracted data. Paper allocation was not random, for example, we deliberately sent translated papers to native languages speakers if available. Comments were returned to the review team who modified the data as required.

\section{Data items extracted}

Information that was extracted from each study was divided into manuscript-level information, farm-level information, piglet information at enrollment, procedure information, intervention information, and outcome information.

Manuscript-level information included the last name of the first author, the year of publication, the original language, and publication type (i.e. published article, conference proceeding, thesis, or report).

Farm-level information included: (1) the region (i.e. North America, South America, Europe, Asia, Oceania, Africa, or not reported), (2) the country, (3) the study setting (i.e. university-owned farm, laboratory or research facility, privately owned/commercial operation, or not reported), (4) the production system (i.e. all-in-allout, continuous flow, unclear, not applicable, or not reported), and (5) sow management (i.e. farrowing crates, free housing, other, unclear, or not reported).

Piglet information at enrollment included: (1) the number of piglets enrolled in the study (including nonrelevant arms), (2) the number of litters represented by the study population, (3) age at enrollment (reported as range, mean and measure of variation, exact number, or not reported), (4) weight at time of procedure (reported as range, mean and measure of variation, exact number, or not reported), (5) breed (meat breed, other, or not reported), (6) the number of relevant trial arms, (7) the types of arms (parallel, non-parallel, or not reported), and (8) the intervention type (pharmacological, nonpharmacological, or other).

Procedure information collected included castration and the technique applied (i.e. scrotal incision and cut of the spermatic cord for removal of testicles or scrotal incision and tear of the spermatic cord for removal of testicles), tail docking and the technique applied (i.e. hot-clipping or cold-clipping), tooth resection and technique applied (i.e. teeth clipped or teeth grinding), and methods of identification that involve cutting of the ear tissue and technique applied (i.e. ear tagging or ear notching).

Intervention information for each group was the type of treatment (i.e. general anesthetic, local anesthetic, NSAID, or comparison to treatment), route of administration, time of administration relative to the procedure, dosage, frequency, the number of animals that received the treatment, and the number of replicates.

For continuous outcomes, we attempted to extract the mean, standard deviation (SD) and/or standard error of the mean, the unit for the measurement, $P$-value, and the number of piglets in the group. If data were reported as medians or quartiles, we extracted the data, although it was not included in meta-analyses nor is it reported here. For categorical outcomes, we attempted to extract the proportion of piglets with the outcome in the group $(r)$, the total number of animals in the group $(n)$, the unit for the of measurement and $P$-value. When an outcome was reported as being measured but no results were reported or data were impossible to extract, we indicated this as 'described but not reported.'

\section{Data manipulations and considerations for data extraction}

Data were converted to standard international (SI) units. An electronic ruler program was used to measure the size of error bars for data extraction from figures on PDF files. When the incomplete outcome data were reported (missing SEM or SD) we used the calculator in Review Manager (RevMan, 2012) provided the design was two-arm and sufficient information as reported for the calculations. When studies reported multiple outcomes within the periods of interest (e.g. measurements of cortisol at $10 \mathrm{~min}, 20 \mathrm{~min}$, and $40 \mathrm{~min}$, all of which would qualify for the under 60-min time period), we used the time point where the comparison group level of the outcome was highest in subsequent meta-analyses.

\section{Risk of bias in individual studies}

We extracted the use of randomization or other restrictions (blocking, stratification) to allocate piglets to groups, where reported. If authors used the term 'random' or 'randomly' to describe assignment to groups, randomization was considered reported. We extracted blinding of outcome assessment.

Only for studies that contributed data to metaanalyses (discussion later), we modified the Cochrane 
Collaboration Risk of Bias Tool (Higgins and Green, 2011) to assess the risk of bias in the individual studies. Only four of the seven risk domains were considered relevant to the review: (1) random sequence generation selection bias, (2) allocation concealment bias - selection bias, (3) blinding of participants and personnel performance bias, and (4) blinding of outcome assessment - detection bias.

\section{Random sequence generation - selection bias}

Studies that did not describe random allocation of piglets to groups were classified as having 'unclear risk' of bias for this risk domain. For studies that described random allocation of piglets to groups, if no description of the random allocation process was provided, then the study was considered to a have an 'unclear risk' of bias. For studies in which a description of random allocation approach was provided (i.e. random number generation or flipping a coin), the risk of bias was assessed as follows:

1. If a simple or restricted random allocation was used and the study size had at least 30 participants, we considered this risk domain to have a low risk of bias.

2. If a simple random allocation was used and the study size was less than 30, we considered this risk domain to have a high risk of bias.

3. If a restricted random allocation was used that accounted for the confounders of age, sow or litter, weight, and adoption (cross-fostering), and if the study size was less than 30, we considered this risk domain to have a low risk of bias.

\section{Allocation concealment - selection bias}

Studies that did not describe random allocation of piglets to groups were classified as having 'unclear risk' of bias. For studies that described random allocation of piglets to groups, we assessed whether allocation concealment was reported. If the method used to conceal the allocation sequence was described in sufficient detail to determine whether intervention allocations could have been foreseen in advance of or during enrollment, we continued to assess the risk of bias as either 'Low risk' of bias, 'High risk' of bias, or 'Unclear risk' of bias based on descriptions by Higgins and Green (2011). If the method used to conceal the allocation sequence was insufficiently described, the study was described as having an 'unclear risk' of bias.

\section{Performance bias}

For the assessment of blinding participants and personnel, we assessed whether the study described measures used to blind caregivers from knowledge of which intervention a participant received and whether the intended blinding was effective. Performance bias was considered only relevant for the long-term outcomes, adverse events: mortality, morbidity, and impaired wound healing. If the method used for caregiver concealment was described in sufficient detail to make a determination of its efficacy, we continued to assess the risk of performance bias as either 'Low risk', 'High risk', or 'Unclear risk' based on descriptions by Higgins and Green (2011). If the method used for caregiver concealment was insufficiently described, the study was described as having an 'unclear risk' of bias.

\section{Detection bias}

The relevance of blinding must be assessed at the outcome level; therefore, we first made judgments about which outcomes were at a 'high risk' or 'low risk' of detection bias if the study was not blinded. We considered that the behavioral outcomes, including vocalisations, were at high risk of bias if blinding was not reported and at low risk of bias if blinding was reported. Physiological and biochemical outcomes were considered to be at low risk of bias regardless of the presence or absence of blinding

\section{Summary measures}

For continuous outcomes the primary summary effect measure was the mean difference or the standardized mean difference between treatment groups when appropriate. For categorical outcomes, the proposed summary effect measure was either the risk or prevalence ratio.

\section{Synthesis of results}

For descriptive purposes, forest plots were created in RevMan (2012). When a study had multiple relevant interventions and one control arm, the control data were used more than once. A random effects model was used. Heterogeneity of the summary effect in each subgroup was tested using Cochran's $Q\left(\chi^{2}\right)$ statistic (significance level $P<0.10$ ). Tau-squared (i.e. the between-study variation) for the subgroups was determined. The $I^{2}$ statistic was also reported. Summary effect measures and 95\% confidence intervals for each intervention-outcome comparison subgroup were reported, regardless of magnitude of heterogeneity measures.

\section{Risk of bias across studies}

The protocol proposed an assessment of publication bias using funnels plots and other statistical methods as appropriate.

\section{Additional analyses}

No additional analyses were performed. 
PRISMA Flow Diagram
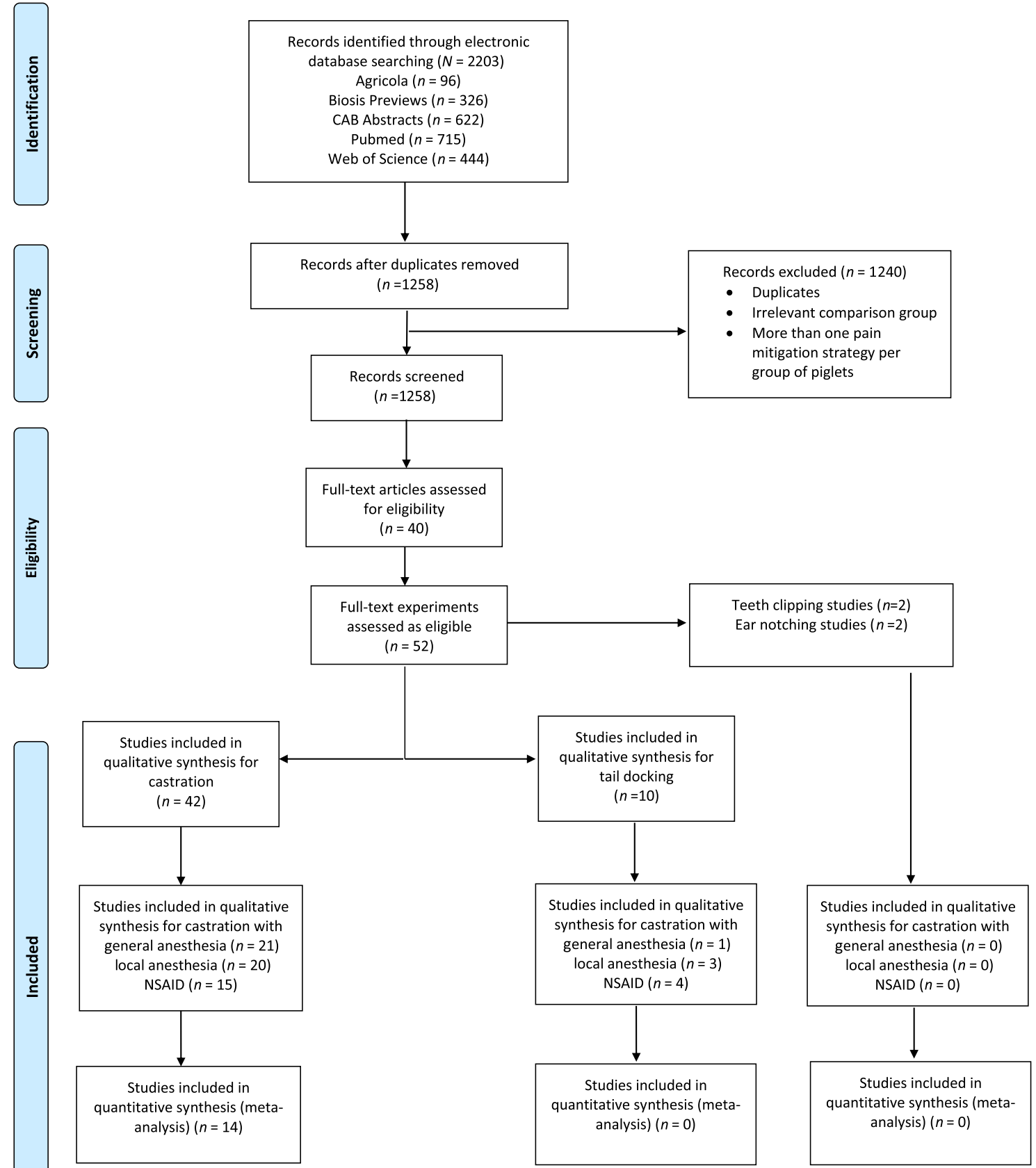

Fig. 1. Study flow diagram.

\section{Results}

\section{Study selection}

The search results are provided in Fig. 1. We searched Google ${ }^{\circledR}$ for electronic versions of these articles before requesting copies through the Iowa State University Library. In this way, we retrieved a thesis that had originally been retrieved as a conference proceeding. The thesis had two chapters with four studies relevant to the review. In the end, 64 full articles were reviewed.
Twenty-four full articles did not meet the eligibility criteria, leaving 40 full articles describing 52 studies included in this review.

\section{Study characteristics}

Summary characteristics of the 52 studies included in the review are shown in Table 3 and characteristics of the 52 individual studies are reported in Table 4 and Figs. 2-6. 
Table 3. Characteristics of relevant experiments identified by the review

\begin{tabular}{|c|c|c|c|c|}
\hline$\underline{\text { Study characteristics }}$ & Castration & Tail docking & Ear notching & Teeth clipping \\
\hline Studies & 42 & 10 & 2 & 2 \\
\hline Technique & - & - & - & - \\
\hline Castration - cut & 21 & - & - & - \\
\hline Castration - tear & 5 & - & - & - \\
\hline Castration - not reported & 16 & - & - & - \\
\hline Tail docking - side cutters & - & 7 & - & - \\
\hline Tail docking - blade & - & 2 & - & - \\
\hline Tail docking - surgical cutters & - & 1 & - & - \\
\hline Ear ID - notching & - & - & 2 & - \\
\hline Teeth trimming - clip & - & - & - & 2 \\
\hline \multicolumn{5}{|l|}{ Language } \\
\hline English & 24 & 8 & 2 & 2 \\
\hline German & 17 & 0 & 0 & 0 \\
\hline French & 2 & 2 & 0 & 0 \\
\hline Danish & 1 & 0 & 0 & 0 \\
\hline \multicolumn{5}{|l|}{ Type of Publication } \\
\hline Published article & 38 & 7 & 2 & 2 \\
\hline Conf. Proceeding & 2 & 0 & 0 & 0 \\
\hline Thesis & 2 & 3 & 0 & 0 \\
\hline Report & 1 & 0 & 0 & 0 \\
\hline Not reported & 1 & 0 & 0 & 0 \\
\hline \multicolumn{5}{|l|}{ Country } \\
\hline USA & 2 & 1 & 0 & 0 \\
\hline Australia & 0 & 2 & 2 & 2 \\
\hline Germany & 8 & 0 & 0 & 0 \\
\hline France & 1 & 2 & 0 & 0 \\
\hline Belgium & 1 & 0 & 0 & 0 \\
\hline Brazil & 1 & 0 & 0 & 0 \\
\hline Switzerland & 2 & 0 & 0 & 0 \\
\hline Sweden & 1 & 0 & 0 & 0 \\
\hline Not explicitly reported & 28 & 5 & 0 & 0 \\
\hline \multicolumn{5}{|l|}{ Setting } \\
\hline University farm & 3 & 2 & 2 & 2 \\
\hline Private farm & 4 & 3 & 0 & 0 \\
\hline Laboratory facility & 4 & 2 & 0 & 0 \\
\hline Not reported & 33 & 3 & 0 & 0 \\
\hline \multicolumn{5}{|l|}{ Production system } \\
\hline All in/all out & 3 & 3 & 0 & 0 \\
\hline Not reported & 41 & 7 & 2 & 2 \\
\hline \multicolumn{5}{|l|}{ Management system } \\
\hline Farrowing crates & 10 & 6 & 0 & 0 \\
\hline Farrowing pens & 1 & 0 & 0 & 0 \\
\hline Individual farrowing pens & 1 & 0 & 0 & 0 \\
\hline Not reported & 32 & 4 & 2 & 2 \\
\hline Total number of animals & 9766 & 5065 & 115 & 117 \\
\hline Not reported (studies) & 2 & 0 & 0 & 0 \\
\hline \multicolumn{5}{|l|}{ Age } \\
\hline Range & 1-28 days & $18 \mathrm{~h}$ to 7 days & 0 & 0 \\
\hline $\operatorname{Max}$ & 28 days & 7 days & 3 days & 3 days \\
\hline Min & 1 day & $18 \mathrm{~h}$ & 0 & 0 \\
\hline Not reported (studies) & 1 & 0 & 0 & 0 \\
\hline \multicolumn{5}{|l|}{ Weight } \\
\hline Range & At least $1-10.6 \mathrm{~kg}$ & 0 & 0 & 0 \\
\hline Max & $10.6 \mathrm{~kg}$ & 0 & 0 & 0 \\
\hline Min & At least $1 \mathrm{~kg}$ & 0 & 0 & 0 \\
\hline \multicolumn{2}{|l|}{ Other } & \multicolumn{3}{|l|}{ Mean $2.2 \pm 0.45$ days } \\
\hline Not reported (studies) & 31 & 8 & 2 & 2 \\
\hline Breed & & & & \\
\hline Meat breed & 31 & 9 & 2 & 2 \\
\hline Not reported & 12 & 1 & 0 & 0 \\
\hline Other & 1 & 0 & 0 & 0 \\
\hline
\end{tabular}


Table 3 (Continued)

\begin{tabular}{|c|c|c|c|c|}
\hline Study characteristics & Castration & Tail docking & Ear notching & Teeth clipping \\
\hline \multicolumn{5}{|c|}{ Number of rel. arms in study } \\
\hline 2 & 23 & 8 & 2 & 2 \\
\hline 3 & 11 & 0 & 0 & 0 \\
\hline 4 & 7 & 0 & 0 & 0 \\
\hline 5 & 2 & 2 & 0 & 0 \\
\hline 6 & 1 & 0 & 0 & 0 \\
\hline \multicolumn{5}{|l|}{ Intervention } \\
\hline Pharmacological & 39 & 5 & 0 & 0 \\
\hline Non-pharmacological & 6 (1 says both) & 7 (2 say both) & 2 & 2 \\
\hline
\end{tabular}

\section{Procedures used}

Piglets in 42 studies underwent castration only. Piglets in two studies underwent both castration and tail docking (Tenbergen, 2012). Piglets in ten studies underwent tail docking only and piglets in two studies underwent ear notching and teeth clipping (Rand et al., 2002). Of the studies in which castration was performed 19 studies assessed general anesthesia protocols, 16 studies assessed local anesthesia protocols, and 14 studies assessed NSAID protocols. Where piglets were tail-docked, two studies assessed general anesthesia, four studies assessed local anesthesia, and three studies assessed NSAIDs. Four studies assessed the pain mitigation efficacy of sucrose after piglets underwent teeth clipping, ear notching, and tail docking (Rand et al., 2002).

\section{Outcomes reported}

The outcomes reported by each study are reported in Table 4. The frequencies of outcomes are provided in Table 5. The most commonly reported outcome was cortisol (18 of 52 studies). Commonly, measures of variation or the actual outcome were not reported, and instead only a $P$-value or the interpretation of the $P$-value was reported. Eighteen studies described adverse events: herniations $(n=1)$, mortality $(n=9)$, impaired wound healing $(n=11)$, and morbidity $(n=2)$. (McGlone et al., 1987; Waldmann et al., 1994; Prunier et al., 2001; Mauch and Bilkei, 2004; Lahrmann et al., 2006; Rittershaus et al., 2009; Sutherland et al., 2010; Hansson et al., 2011; Rault and Lay, 2011; Sutherland et al., 2011; Reiner et al., 2012; Schwab et al., 2012; Sutherland et al., 2012; Tenbergen, 2012).

\section{Risk of bias within relevant studies}

Randomization, blinding, and blocking are described in Table 4. Thirty-three studies used the term 'randomly', 'randomized', or 'random' in their description of piglet allocation to groups. Restriction of randomization by either blocking by continuous covariates or stratification by categorical covariates was reported in 39 studies. No study that controlled for continuous outcomes explicitly reported the block size. Eighteen studies reported blinding, but did not provide a full description of the approach used to blind the study or the task was blinded.

With respect to the risk of selection bias in those studies eventually included meta-analyses (listed below), none provided sufficient detail of random sequence generation or allocation concealment to assess the potential for risk. Therefore, in this group of studies, the risk of selection bias on the efficacy of the interventions is unclear. The risk of performance bias was considered relevant for adverse events - herniations, impaired wound healing, morbidity. However, this risk domain could not be assessed because adverse outcomes were poorly reported and caregiver blinding was never reported. The approach to blinding of outcome assessors and its effectiveness was not reported or insufficiently reported, making the risk of detection bias unclear for all outcomes in the metaanalysis.

\section{Results of individual studies}

As reported the accumulated data were sparse and although many studies reported measuring outcomes, frequently data were reported in a manner that precluded extraction. As a result it was decided by the review panel to conduct meta-analysis for seven outcomes:

1. Cortisol;

2. Norepinephrine;

3. $\beta$-endorphins;

4. Frequency of vocalisations was described using the units hertz $(\mathrm{Hz})$;

5. Energy or pitch of vocalisations were described using the units decibels $(\mathrm{dB})$;

6. Vocalisation rate or risk was measured, such as the number of piglets that vocalized after castration per unit of time or the number of animals that vocalized from the group;

7. Pain-related behaviours - this outcome was defined after data extraction.

This approach to synthesis was based on discussions with the content experts after data extraction and assessment of data availability. The rationale for presenting these 
Table 4. Characteristics of studies included in the review

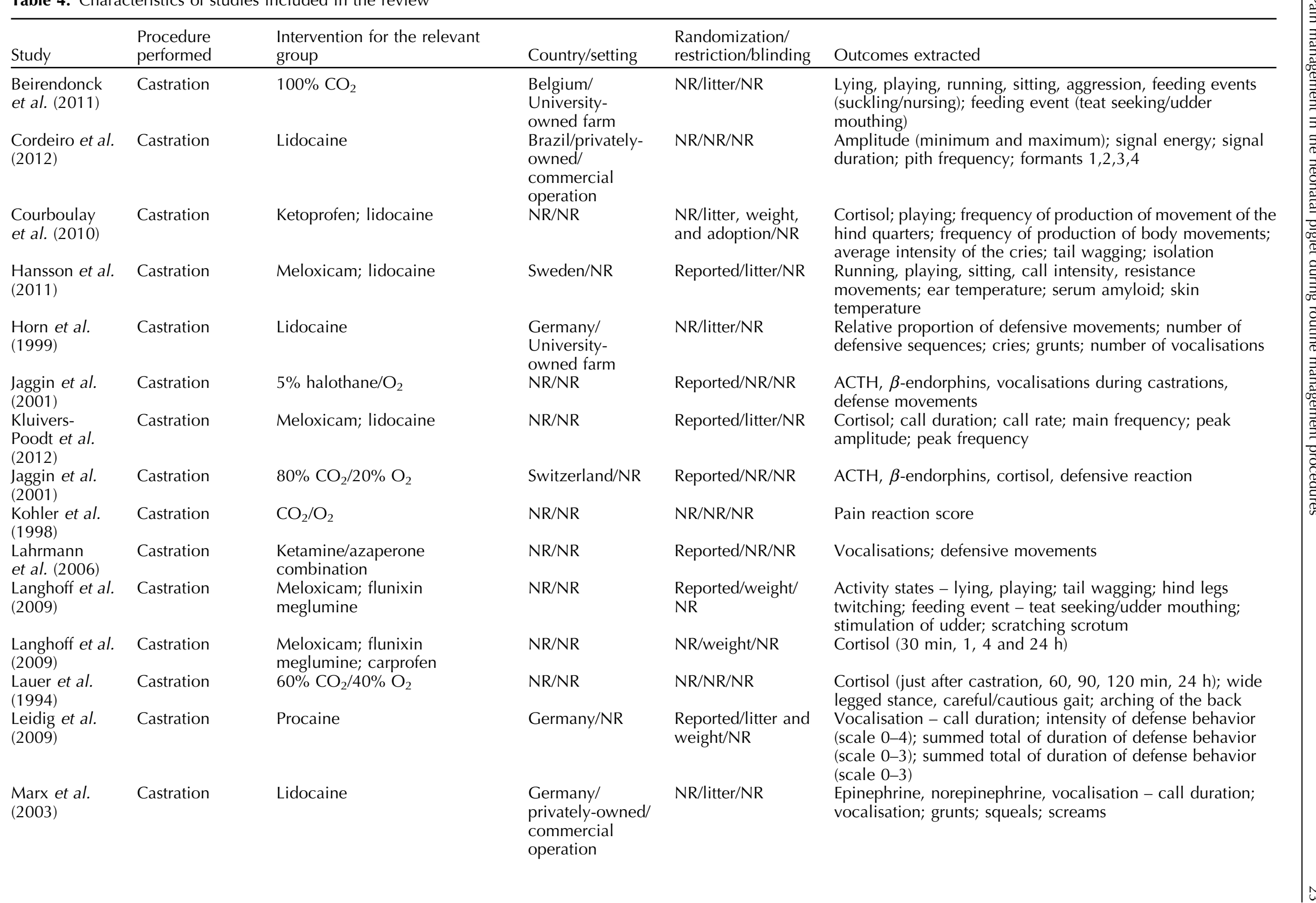




\begin{tabular}{|c|c|c|c|c|c|}
\hline Study & $\begin{array}{l}\text { Procedure } \\
\text { performed }\end{array}$ & $\begin{array}{l}\text { Intervention for the relevant } \\
\text { group }\end{array}$ & Country/setting & $\begin{array}{l}\text { Randomization/ } \\
\text { restriction/blinding }\end{array}$ & Outcomes extracted \\
\hline $\begin{array}{l}\text { Mauch and } \\
\text { Bilkei (2004) }\end{array}$ & Castration & $\begin{array}{l}\text { Acepromazine and ketamine; } \\
\text { azaperone and ketamine; } \\
\text { acepromazine and } 10 \% \\
\text { ketamine }\end{array}$ & NR/NR & NR/litter/NR & Post-surgical anesthesia/surgical sleep \\
\hline $\begin{array}{l}\text { McGlone } \\
\text { et al. (1987) }\end{array}$ & Castration & $\begin{array}{l}\text { Xylazine, ketamine and } 5 \% \\
\text { glyceryl guaiacolate }\end{array}$ & NR/NR & $\begin{array}{l}\text { Reported/NR/ } \\
\text { reported }\end{array}$ & $\begin{array}{l}\text { \# missed nursing, standing under heat, lying under heat, } \\
\text { standing away from heat, lying away from heat, nursing, } \\
\text { incoordinated }\end{array}$ \\
\hline $\begin{array}{l}\text { McGlone } \\
\text { et al. (1987) }\end{array}$ & Castration & Lidocaine & NR/NR & $\begin{array}{l}\text { Reported/NR/ } \\
\text { reported }\end{array}$ & $\begin{array}{l}\text { Standing under heat, lying under heat, standing away from } \\
\text { heat, lying away from heat, nursing, incoordinated }\end{array}$ \\
\hline $\begin{array}{l}\text { Muhlbauer } \\
\text { et al. (2009) }\end{array}$ & Castration & $70 \% \mathrm{CO}_{2} / 30 \% \mathrm{O}_{2}$ & $\begin{array}{l}\text { Germany/ } \\
\text { laboratory/ } \\
\text { research facility }\end{array}$ & $\begin{array}{l}\text { Reported/weight/ } \\
\text { NR }\end{array}$ & $\begin{array}{l}\text { Cortisol (30 min, } 1,4 \mathrm{~h} \text { and } 1 \mathrm{~d}) \text {; epinephrine }(2 \mathrm{~min}) \text {; } \\
\text { norepinephrine }(2 \mathrm{~min})\end{array}$ \\
\hline $\begin{array}{l}\text { Nyborg et al. } \\
(2000)\end{array}$ & Castration & $\begin{array}{l}\text { Bupivacaine (with } \\
\text { noradrenaline) }\end{array}$ & NR/NR & $\begin{array}{l}\text { Reported/NR/ } \\
\text { reported }\end{array}$ & Squealing; no. of c-Fos-positive neurons \\
\hline $\begin{array}{l}\text { Prunier et al. } \\
\text { (2001) }\end{array}$ & Tail docking & $\begin{array}{l}\text { Vaporizing of an instant } \\
\text { cry-active product }\end{array}$ & $\begin{array}{l}\text { France/ } \\
\text { laboratory/ } \\
\text { research facility }\end{array}$ & NR/litter/NR & Lying, sitting, udder mouthing \\
\hline $\begin{array}{l}\text { Prunier et al. } \\
(2001)\end{array}$ & Tail docking & Vaporized refrigerant & $\begin{array}{l}\text { France/ } \\
\text { laboratory/ } \\
\text { research facility }\end{array}$ & NR/litter/NR & ACTH, cortisol \\
\hline $\begin{array}{l}\text { Rand et al. } \\
(2002)\end{array}$ & Ear notching & Sucrose & $\begin{array}{l}\text { Australia/ } \\
\text { University- } \\
\text { owned farm }\end{array}$ & $\begin{array}{l}\text { Reported/litter/ } \\
\text { reported }\end{array}$ & Tail flicking, tail jamming, grunt, squeal \\
\hline $\begin{array}{l}\text { Rand et al. } \\
(2002)\end{array}$ & $\begin{array}{l}\text { Tail docking, } \\
\text { teeth clipping, } \\
\text { and ear } \\
\text { notching }\end{array}$ & Sucrose & $\begin{array}{l}\text { Australia/ } \\
\text { University- } \\
\text { owned farm }\end{array}$ & $\begin{array}{l}\text { Reported/litter/ } \\
\text { reported }\end{array}$ & Tail flicking, tail jamming, grunt, squeal \\
\hline $\begin{array}{l}\text { Rand et al. } \\
(2002)\end{array}$ & Tail docking & Sucrose & $\begin{array}{l}\text { Australia/ } \\
\text { University- } \\
\text { owned farm }\end{array}$ & $\begin{array}{l}\text { Reported/litter/ } \\
\text { reported }\end{array}$ & Head shaking, tail flicking, tail jamming \\
\hline $\begin{array}{l}\text { Rand et al. } \\
(2002)\end{array}$ & Teeth clipping & Sucrose & $\begin{array}{l}\text { Australia/ } \\
\text { University- } \\
\text { owned farm }\end{array}$ & $\begin{array}{l}\text { Reported/litter/ } \\
\text { reported }\end{array}$ & Tail flicking, tail jamming, grunt, squeal \\
\hline $\begin{array}{l}\text { Rault and Lay } \\
\text { (2011) }\end{array}$ & Castration & $\mathrm{N}_{2} \mathrm{O} / \mathrm{O}_{2}$ & $\begin{array}{l}\text { USA/laboratory/ } \\
\text { research facility }\end{array}$ & $\begin{array}{l}\text { NR/litter and } \\
\text { weight/reported }\end{array}$ & $\begin{array}{l}\text { Tail wag, scratching quarter, agitation frequency, agitation } \\
\text { intensity, huddle up, Vocalisation (both high frequency [i.e. } \\
\text { squeals] and low frequency [i.e. grunts]) length during } \\
\text { castration }\end{array}$ \\
\hline $\begin{array}{l}\text { Reiner et al. } \\
(2012)\end{array}$ & Castration & Flunixin; flunixin; meloxicam & Germany/NR & $\begin{array}{l}\text { Reported/litter and } \\
\text { weight/reported }\end{array}$ & $\begin{array}{l}\text { Cortisol; stress vocalisations; suckling/nursing; teat seeking/ } \\
\text { udder mouthing; teat seeking/udder mouthing; walking; } \\
\text { flinching }\end{array}$ \\
\hline $\begin{array}{l}\text { Rittershaus } \\
\text { et al. (2009) }\end{array}$ & Castration & $\begin{array}{l}\text { Chorethyl cooling spray; } \\
\text { EMLA-cream }\end{array}$ & $\begin{array}{l}\text { Germany/ } \\
\text { laboratory/ } \\
\text { research facility }\end{array}$ & $N R / N R / N R$ & $\begin{array}{l}\text { Cortisol ( } 1 \text { and } 24 \mathrm{~h}) \text {; scream frequency; high frequency; } \\
\text { entropy and duration }\end{array}$ \\
\hline
\end{tabular}




\begin{tabular}{|c|c|c|c|c|c|}
\hline Study & $\begin{array}{l}\text { Procedure } \\
\text { performed }\end{array}$ & $\begin{array}{l}\text { Intervention for the relevant } \\
\text { group }\end{array}$ & Country/setting & $\begin{array}{l}\text { Randomization/ } \\
\text { restriction/blinding }\end{array}$ & Outcomes extracted \\
\hline $\begin{array}{l}\text { Rutherford } \\
\text { et al. (2009) }\end{array}$ & Tail docking & $\begin{array}{l}\text { Prenatal stress of sows - } \\
\text { mingled twice during gestation }\end{array}$ & NR/NR & NR/sow/reported & Pain scores \\
\hline $\begin{array}{l}\text { Schmidt et al. } \\
\text { (2012) }\end{array}$ & Castration & Meloxicam & $\begin{array}{l}\text { Germany/ } \\
\text { University- } \\
\text { owned farm }\end{array}$ & $\begin{array}{l}\text { Reported/litter and } \\
\text { weight/reported }\end{array}$ & $\begin{array}{l}\text { Standing, walking; Feeding event - teat seeking/udder } \\
\text { mouthing; Feeding state - teeth champing/chewing }\end{array}$ \\
\hline $\begin{array}{l}\text { Schonreiter } \\
\text { et al. (2000) }\end{array}$ & Castration & $\mathrm{CO}_{2} / \mathrm{O}_{2}$ & NR/NR & NR/NR/NR & $\begin{array}{l}\text { Cortisol ( } 1 \text { and } 24 \mathrm{~h} \text { ); } \beta \text {-endorphins ( } 1 \text { and } 24 \text { h); (playing, } \\
\text { suckling, standing) }\end{array}$ \\
\hline $\begin{array}{l}\text { Schulz et al. } \\
(2007 \mathrm{~b})\end{array}$ & Castration & $5 \%$ isoflurane & NR/NR & Reported/NR & Epinephrine and norepinephrine (just after castration) \\
\hline $\begin{array}{l}\text { Schulz et al. } \\
\text { (2007a) }\end{array}$ & Castration & $5 \%$ isoflurane & $N R / N R$ & Reported/NR/NR & Cortisol (at $30 \mathrm{~min}, 1,4$ and $24 \mathrm{~h}$ ) \\
\hline $\begin{array}{l}\text { Schwab et al. } \\
(2012)\end{array}$ & Castration & Ketoprofen & $\begin{array}{l}\text { France/ } \\
\text { laboratory/ } \\
\text { research facility }\end{array}$ & Reported/litter/NR & ACTH; Cortisol; mean behavior score $(1,2,4$ and $24 \mathrm{~h})$ \\
\hline $\begin{array}{l}\text { Sutherland } \\
\text { et al. (2010) }\end{array}$ & Castration & Flunixin meglumine & NR/NR & $\begin{array}{l}\text { Reported/litter and } \\
\text { weight/reported }\end{array}$ & $\begin{array}{l}\text { Cortisol (30, 60, 120, } 180 \mathrm{~min}) \text {; substance } \mathrm{P}(30,60 \text {, } \\
120 \mathrm{~min}) \text {; C-reactive protein }\end{array}$ \\
\hline $\begin{array}{l}\text { Sutherland } \\
\text { et al. (2011) }\end{array}$ & Tail docking & $\begin{array}{l}100 \% \mathrm{CO}_{2} \text {; lidocaine; } \\
\text { cetacaine; tri-solfen }\end{array}$ & NR/NR & $\begin{array}{l}\text { Reported/litter and } \\
\text { weight/NR }\end{array}$ & Cortisol $(30,60,90 \mathrm{~min}$ and $4 \mathrm{~h})$ \\
\hline $\begin{array}{l}\text { Sutherland } \\
\text { et al. (2012) }\end{array}$ & Castration & $100 \% \mathrm{CO}_{2} ;$ flunixin meglumine & NR/NR & $\begin{array}{l}\text { Reported/litter and } \\
\text { weight/NR }\end{array}$ & $\begin{array}{l}\text { Lying without contact; lying with contact; stress vocalisations; } \\
\text { pain-like behavior, walking, standing, sitting }\end{array}$ \\
\hline $\begin{array}{l}\text { Sutherland } \\
\text { et al. (2010) }\end{array}$ & Castration & Flunixin meglumine & NR/NR & $\begin{array}{l}\text { Reported/litter and } \\
\text { weight/reported }\end{array}$ & $\begin{array}{l}\text { Lying without contact; lying with contact; stress vocalisations; } \\
\text { pain-like behavior, walking, standing, sitting }\end{array}$ \\
\hline $\begin{array}{l}\text { Sutherland } \\
\text { et al. (2011) }\end{array}$ & Tail docking & $\begin{array}{l}100 \% \mathrm{CO}_{2} ; \text { lidocaine; } \\
\text { cetacaine; Tri-solfen }\end{array}$ & $N R / N R$ & $\begin{array}{l}\text { Reported/litter and } \\
\text { weight/NR }\end{array}$ & $\begin{array}{l}\text { Vocalisation, suckling, standing, sitting, walking, pain-like } \\
\text { behaviors, active }\end{array}$ \\
\hline $\begin{array}{l}\text { Sutherland } \\
\text { et al. (2012) }\end{array}$ & Castration & $100 \% \mathrm{CO}_{2} ;$ flunixin meglumine & NR/NR & $\begin{array}{l}\text { Reported/litter and } \\
\text { weight/NR }\end{array}$ & $\begin{array}{l}\text { Cortisol }(30,60,120,180 \text { min, } 24 \text { h); Substance P }(30,60 \text {, } \\
120,180 \mathrm{~min}) \text {; C-reactive protein }\end{array}$ \\
\hline $\begin{array}{l}\text { Tenbergen } \\
\text { (2012) }\end{array}$ & $\begin{array}{l}\text { Castration, tail } \\
\text { docking }\end{array}$ & Meloxicam & NR/NR & $\begin{array}{l}\text { Reported/litter/ } \\
\text { reported }\end{array}$ & Cortisol $(30,60,90 \mathrm{~min}$ and $4 \mathrm{~h})$ \\
\hline $\begin{array}{l}\text { Tenbergen } \\
(2012)\end{array}$ & $\begin{array}{l}\text { Castration, tail } \\
\text { docking }\end{array}$ & Ketoprofen & NR/NR & $\begin{array}{l}\text { Reported/litter/ } \\
\text { reported }\end{array}$ & Cortisol $(30,60,90 \mathrm{~min}$ and $4 \mathrm{~h})$ \\
\hline $\begin{array}{l}\text { Tenbergen } \\
(2012)\end{array}$ & Tail docking & Ketoprofen & $\begin{array}{l}\text { NR/privately- } \\
\text { owned/ } \\
\text { commercial } \\
\text { operation }\end{array}$ & $\begin{array}{l}\text { Reported/litter/ } \\
\text { reported }\end{array}$ & Cortisol $(30,60,90 \mathrm{~min}$ and $4 \mathrm{~h})$ \\
\hline $\begin{array}{l}\text { Waldmann } \\
\text { et al. (1994) }\end{array}$ & Castration & $\begin{array}{l}\text { Butanilicaine phosphate; } \\
\text { thiopental sodium tiletamine/ } \\
\text { zolazepam; propofol }\end{array}$ & NR/NR & NR/litter NR & Rectal temperature (30 min, 1, 3 and $5 \mathrm{~h}$ ) \\
\hline $\begin{array}{l}\text { Walker et al. } \\
(2004)\end{array}$ & Castration & $\begin{array}{l}\text { Isoflurane } / \mathrm{O}_{2} ; \text { isoflurane } / \mathrm{N}_{2} \mathrm{O} / \\
\mathrm{O}_{2}\end{array}$ & Switzerland/NR & $\begin{array}{l}\text { NR/litter and } \\
\text { weight/NR }\end{array}$ & $\mathrm{ACTH}, \beta$-endorphins, pain scores \\
\hline $\begin{array}{l}\text { Wavreille } \\
\text { et al. (2012) }\end{array}$ & Castration & Meloxicam; tolfenamic acid & NR/NR & $\begin{array}{l}\text { Reported/weight/ } \\
\text { reported }\end{array}$ & $\begin{array}{l}\text { Cortisol; heart rate; scratching of scrotum; trembling or } \\
\text { spasms; crying; isolation }\end{array}$ \\
\hline $\begin{array}{l}\text { White et al. } \\
\text { (1995) }\end{array}$ & Castration & Lidocaine & NR/NR & NR/litter/NR & $\begin{array}{l}\text { Heart rate, respiratory rate; mean frequency with the highest } \\
\text { energy (HEF) }\end{array}$ \\
\hline
\end{tabular}


outcomes was that: (1) these outcomes were ranked as important in a separate exercise used for the recommendation-making project (results not reported), and (2) data were available for these outcomes. For presentation purposes, the intervention types were organized as general anesthetic, local anesthetic, and NSAID and presented within those categories. Finally, as the objective of the study was to summarize bodies of work rather than rewrite single study results, further summarization was only conducted on outcome-intervention-procedure combinations with more than one independent study reported in a manner that enabled calculation of an effect measure. The absence of a forest plot for a procedureintervention combination therefore implies that either no study reported the outcome or multiple studies reported the outcome, but not more than one in a manner that data could be extracted fully.

\section{Individual study results for castration and general anesthetic protocols}

Figures 2 and 3 are forest plots that include, in the columns on the center side of the figure mean of cortisol, the SD and number of animals enrolled each trial arm for piglets that received general anesthesia prior to castration under $60 \mathrm{~min}$ and between 1 and $24 \mathrm{~h}$, respectively. Although many studies reported cortisol, only three studies (Kohler et al., 1998; Schonreiter et al., 2000; Muhlbauer et al., 2009) provided complete information.

Figure 4 is a forest plot that presents the study level data for blood $\beta$-endorphin levels under $60 \mathrm{~min}$ for studies that assessed the pain mitigation efficacy of isoflurane, halothane, and $\mathrm{CO}_{2} / \mathrm{O}_{2}$ anesthesia. For isoflurane, only a single article with three trial arms was available, so no summary calculation was possible. There appeared to be no significant difference in blood $\beta$-endorphin levels under $60 \mathrm{~min}$ in piglets that were castrated while receiving isoflurane (MD: 1.30; 95\% CI: -3.30-5.98; $P=0.59$ ), halothane (MD: 1.59; 95\% CI: $-0.11-3.30$; $P=0.07$ ), or $\mathrm{CO}_{2} / \mathrm{O}_{2}$ anesthesia (MD: 1.06; 95\% CI: -0.66-2.78; $P=0.23$ ), compared with piglets receiving no anesthesia. Although several studies assessed norepinephrine, none provided a comprehensive report; therefore, individual results are not presented. For vocalisation outcomes and pain-related behavioral outcomes, the results of individual studies are reported in Tables 6 and 7. Vocalisation outcomes were often measured at the time of the procedure. Rault and Lay (2011) assessed highfrequency (i.e. squeals) and low-frequency (i.e. grunts) vocalisations during $\mathrm{N}_{2} \mathrm{O} / \mathrm{O}_{2}$ anesthesia and found that 'the treatments did not differ during castration itself ( $P=0.87$ ), with piglets vocalizing $80 \%$ of the time' (Rault and Lay, 2011).

\section{Individual study results for castration and local anesthetic protocols}

Thirteen studies assessed local anesthesia protocols that evaluated cortisol, norepinephrine, or pain-related 


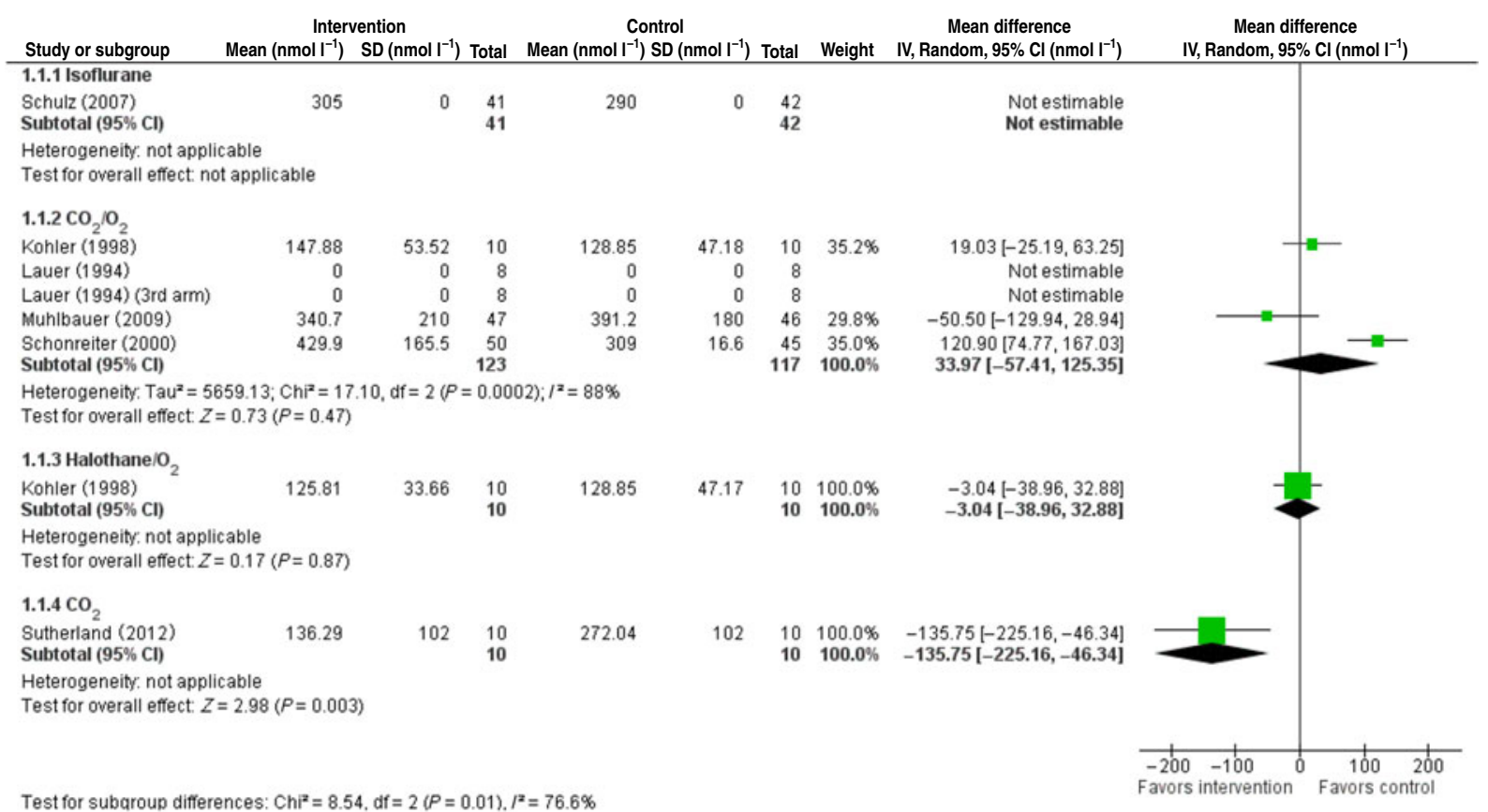

Fig. 2. Forest plot of cortisol $\left(\mathrm{nmol} \mathrm{I} \mathrm{I}^{-1}\right)$ measurements occurring within 60 min of castration for trials comparing the pain responses of piglets that received a variety of general anesthetic to no anesthetic.

\begin{tabular}{|c|c|c|c|c|c|c|c|c|c|c|}
\hline \multirow{2}{*}{$\begin{array}{l}\text { Study or subgroup } \\
1.2 .1 \text { Is oflurane }\end{array}$} & \multicolumn{3}{|c|}{ Intervention } & \multicolumn{3}{|c|}{ Control } & \multirow[t]{2}{*}{ Weight } & \multirow[t]{2}{*}{$\begin{array}{c}\text { Mean difference } \\
\left.\text { IV, Random, } 95 \% \mathrm{Cl}_{(\mathrm{nmol} \mathrm{I}}{ }^{-1}\right)\end{array}$} & \multicolumn{2}{|c|}{$\begin{array}{c}\text { Mean difference } \\
\left.\text { IV, Random, } 95 \% \mathrm{Cl}_{(\mathrm{nmol} \mathrm{I}}^{-1}\right)\end{array}$} \\
\hline & & & & & & & & & & \\
\hline $\begin{array}{l}\text { Schulz (2007) } \\
\text { Subtotal (95\% Cl) }\end{array}$ & 60 & 0 & $\begin{array}{l}41 \\
41\end{array}$ & 70 & 0 & $\begin{array}{l}42 \\
42\end{array}$ & & $\begin{array}{l}\text { Not estimable } \\
\text { Not estimable }\end{array}$ & & \\
\hline \multicolumn{11}{|c|}{$\begin{array}{l}\text { Heterogeneity. not applicable } \\
\text { Test for overall effect: not applicable }\end{array}$} \\
\hline \multicolumn{11}{|l|}{$1.2 .2 \mathrm{CO}_{2} / \mathrm{O}_{2}$} \\
\hline Lauer (1994) & 0 & 0 & 8 & 0 & 0 & 8 & & Not estimable & & \\
\hline Lauer (1994) (3rd arm) & 0 & 0 & 8 & 0 & 0 & 8 & & Not estimable & & \\
\hline Muhlbauer (2009) & 120 & 80 & 47 & 160 & 150 & 46 & $41.3 \%$ & $-40.00[-89.01,9.01]$ & | & \\
\hline $\begin{array}{l}\text { Schonreiter }(2000) \\
\text { Subtotal }(95 \% \mathrm{Cl})\end{array}$ & 126 & 87.8 & $\begin{array}{r}50 \\
113\end{array}$ & 200 & 110 & $\begin{array}{r}45 \\
107\end{array}$ & $\begin{array}{r}58.7 \% \\
100.0 \%\end{array}$ & $\begin{array}{r}-74.00[-114.31,-33.69] \\
-59.97[-92.78,-27.17]\end{array}$ & & \\
\hline \multicolumn{11}{|c|}{$\begin{array}{l}\text { Heterogeneity. } \text { Tau }^{2}=53.82 ; \mathrm{Ch}^{2}=1.10, \mathrm{df}=1(P=0.29) ; /^{2}=9 \% \\
\text { Test for overall effect } Z=3.58(P=0.0003)\end{array}$} \\
\hline \multicolumn{11}{|l|}{$1.2 .3 \mathrm{CO}_{2}$} \\
\hline $\begin{array}{l}\text { Sutherland (2012) } \\
\text { Subtotal (95\% Cl) }\end{array}$ & 40.83 & 108.83 & $\begin{array}{l}10 \\
10\end{array}$ & 126 & 104.1 & $\begin{array}{l}10 \\
10\end{array}$ & $\begin{array}{l}100.0 \% \\
\mathbf{1 0 0 . 0 \%}\end{array}$ & $\begin{array}{l}-85.17[-178.51,8.17] \\
-85.17[-\mathbf{1 7 8 . 5 1}, \mathbf{8 . 1 7}]\end{array}$ & & \\
\hline \multicolumn{11}{|c|}{$\begin{array}{l}\text { Heterogeneity, not applicable } \\
\text { Test for overall effect: } Z=1.79(P=0.07)\end{array}$} \\
\hline Test for subaroun differe & ences: $\mathrm{Chi}^{2}=0.2$ & 25. $\mathrm{df}=1(P=\mathrm{f}$ & $0.62), 1$ & $\rho^{2}=0 \%$ & & & & & $\begin{array}{l}-200-100 \\
\text { Favors intervention }\end{array}$ & $\begin{array}{c}100 \quad 200 \\
\text { Favors control }\end{array}$ \\
\hline
\end{tabular}

Fig. 3. Forest plot of cortisol measurements $\left(\mathrm{nmol} \mathrm{I}^{-1}\right.$ ) occurring between 1 and $24 \mathrm{~h}$ of castration for trials comparing the pain responses of piglets that received a variety of general anesthetic to no anesthetic.

behavior metrics. The local anesthetics assessed included lidocaine, procaine, cetacaine, bupivacaine, and trisolfen. None provided complete reporting for any of the outcomes; therefore, forest plots were not created and individual results (which were incomplete) are not presented. Tables 6 and 7 contain the results for studies that report vocalisation and pain behaviours outcomes for piglets castrated and illustrate the incomplete reporting.

\section{Individual study results for castration and NSAID protocols}

Figure 5 provides the individual results for protocols that assessed the impact of use of NSAIDs on blood cortisol levels under $60 \mathrm{~min}$ for piglets that received meloxicam, ketoprofen, or flunixin meglumine. Three studies (Langhoff et al., 2009; Reiner et al., 2012; Tenbergen, 2012) provided data for the assessment of meloxicam as a pain mitigation strategy. No studies comprehensively reported 


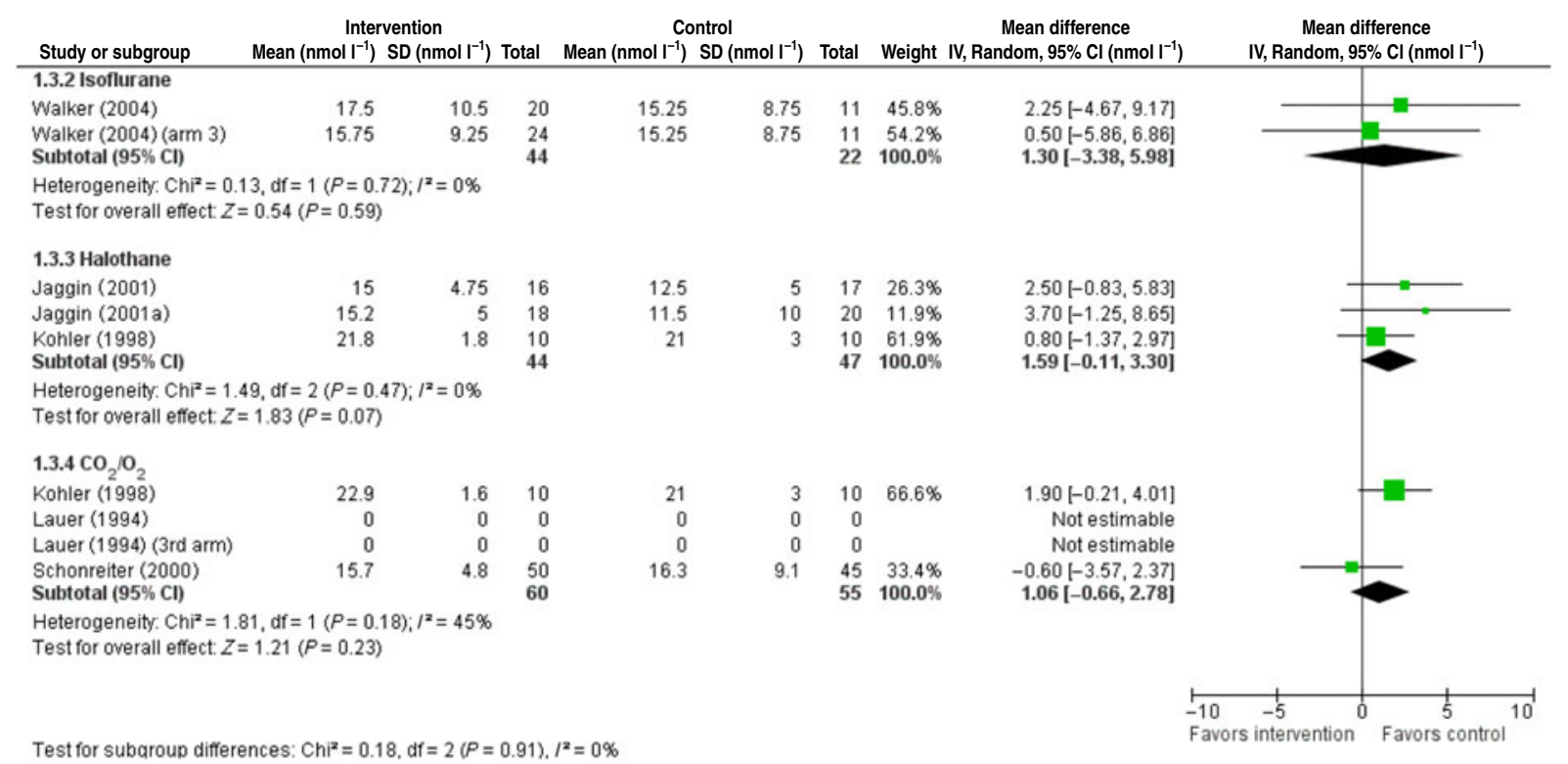

Fig. 4. Forest plot of $\beta$-endorphin measurements $\left(\mathrm{pmol} \mathrm{I}^{-1}\right.$ ) occurring within $60 \mathrm{~min}$ of castration for trials comparing the pain responses of piglets that received a variety of general anesthetic to no anesthetic.

\begin{tabular}{|c|c|c|c|c|c|c|c|c|c|c|}
\hline \multirow{2}{*}{$\begin{array}{l}\text { Study or subgroup } \\
\text { 1.1.1 Meloxicam }\end{array}$} & \multicolumn{3}{|c|}{ Intervention } & \multicolumn{3}{|c|}{ Control } & \multirow[t]{2}{*}{ Weight } & \multirow[t]{2}{*}{$\begin{array}{c}\text { Mean difference } \\
\left.\text { IV, Random, } 95 \% \mathrm{Cl}_{(\mathrm{nmol}} \mathrm{I}^{-1}\right)\end{array}$} & \multicolumn{2}{|c|}{$\begin{array}{c}\text { Mean difference } \\
\mathrm{IV}, \text { Random, } 95 \% \mathrm{Cl}\left(\mathrm{nmol} \mathrm{I}{ }^{-1}\right)\end{array}$} \\
\hline & & & & & & & & & & \\
\hline Langhoff (2009) & 155 & 51 & 25 & 215.4 & 59 & 28 & $45.5 \%$ & $-60.40[-90.02,-30.78]$ & 를 & \\
\hline Reiner (2012) & 291 & 124 & 30 & 354 & 201 & 30 & $27.5 \%$ & $-63.00[-147.51,21.51]$ & & \\
\hline Tenbergen (2012b) & 169.4 & 50.8 & 12 & 344.4 & 150 & 13 & $27.0 \%$ & $-175.00[-261.46,-88.54]$ & $\longrightarrow$ & \\
\hline Wavreille (2012)(\%) & 0 & 0 & 15 & 0 & 0 & 14 & & Not estimable & & \\
\hline Zols $(2006)$ & 95 & 0 & 72 & 210 & 0 & 45 & & Not estimable & & \\
\hline Subtotal $(95 \% \mathrm{Cl})$ & & & 154 & & & 130 & $100.0 \%$ & $-92.02[-158.12,-25.92]$ & & \\
\hline \multicolumn{11}{|c|}{$\begin{array}{l}\text { Heterogeneity. Tau }{ }^{2}=2271.27 ; \mathrm{Chi}^{2}=6.08, \mathrm{df}=2(P=0.05) ; /^{2}=67 \% \\
\text { Test for overall effect. } Z=2.73(P=0.006)\end{array}$} \\
\hline \multicolumn{11}{|l|}{ 1.1.2 Tolfenamic acid } \\
\hline $\begin{array}{l}\text { Wavreille (2012) (9) } \\
\text { Subtotal }(95 \% \mathrm{Cl})\end{array}$ & 551.8 & 110.36 & $\begin{array}{l}17 \\
17\end{array}$ & 0 & 0 & $\begin{array}{l}14 \\
14\end{array}$ & & $\begin{array}{l}\text { Not estimable } \\
\text { Not estimable }\end{array}$ & & \\
\hline \multicolumn{11}{|c|}{$\begin{array}{l}\text { Heterogeneity. not applicable } \\
\text { Test for overall effect. not applicable }\end{array}$} \\
\hline \multicolumn{11}{|l|}{ 1.1.3 Ketopr ofen } \\
\hline Courboulay (2010) & 353.1 & 140.1 & 0 & 540.48 & 0 & 0 & & Not estimable & & \\
\hline Schwab (2012) & 426.6 & 63.9 & 15 & 668 & 66.1 & 15 & $87.2 \%$ & $-241.40[-287.93,-194.87]$ & & \\
\hline $\begin{array}{l}\text { Tenbergen }(2012 \mathrm{c}) \\
\text { Subtotal }(95 \% \mathrm{Cl})\end{array}$ & 178.2 & 104 & $\begin{array}{l}15 \\
30\end{array}$ & 370.3 & 215.7 & $\begin{array}{l}15 \\
30\end{array}$ & $\begin{array}{r}12.8 \% \\
\mathbf{1 0 0 . 0 \%}\end{array}$ & $\begin{array}{r}-192.10[-313.28,-70.92] \\
-235.07[-278.50,-191.63]\end{array}$ & & \\
\hline \multicolumn{11}{|c|}{$\begin{array}{l}\text { Heterogeneity. Tau } \mathrm{T}^{2}=0.00 ; \mathrm{Chi}^{2}=0.55, \mathrm{df}=1(P=0.46) ; I^{2}=0 \% \\
\text { Test for overall effect: } Z=10.61(P<0.00001)\end{array}$} \\
\hline \multicolumn{11}{|c|}{ 1.1.4 Flunixin meglumine } \\
\hline Langhoff (2009) & 138 & 66 & 26 & 215.4 & 59 & 28 & $67.6 \%$ & $-77.40[-110.88,-43.92]$ & & \\
\hline Reiner (3rd arm) & 254 & 124 & 30 & 354 & 201 & 30 & $10.6 \%$ & $-100.00[-184.51,-15.49]$ & & \\
\hline Reiner (2012) & 268 & 87 & 30 & 354 & 201 & 30 & $12.3 \%$ & $-86.00[-164.37,-7.63]$ & & \\
\hline $\begin{array}{l}\text { Sutherland (2012) } \\
\text { Subtotal }(95 \% \mathrm{Cl})\end{array}$ & 220.4 & 102.1 & $\begin{array}{l}10 \\
96\end{array}$ & 272.04 & 102.9 & $\begin{array}{l}10 \\
98\end{array}$ & $\begin{array}{r}9.4 \% \\
100.0 \%\end{array}$ & $\begin{array}{r}-51.64[-141.48,38.20] \\
-78.44[-105.98,-50.90]\end{array}$ & & \\
\hline \multicolumn{11}{|c|}{$\begin{array}{l}\text { Heterogeneity. } \mathrm{Tau}^{2}=0.00 ; \mathrm{Ch}^{2}=0.63, \mathrm{df}=3(P=0.89) ; /^{2}=0 \% \\
\text { Test for overall effect: } Z=5.58(P<0.00001)\end{array}$} \\
\hline \multicolumn{11}{|l|}{ 1.1.5 Carprofen } \\
\hline $\begin{array}{l}\text { Langhoff (2009) } \\
\text { Subtotal }(95 \% \mathrm{Cl})\end{array}$ & 200 & 77 & $\begin{array}{l}15 \\
15\end{array}$ & 215.4 & 59 & $\begin{array}{l}28 \\
28\end{array}$ & $\begin{array}{l}100.0 \% \\
\mathbf{1 0 0 . 0 \%}\end{array}$ & $\begin{array}{l}-15.40[-60.08,29.28] \\
-15.40[-60.08,29.28]\end{array}$ & & \\
\hline \multicolumn{11}{|c|}{$\begin{array}{l}\text { Heterogeneity. not applicable } \\
\text { Test for overall effect: } Z=0.68(P=0.50)\end{array}$} \\
\hline st for subrroun dif & & & & & & & & & $\begin{array}{l}-200-100 \\
\text { ors intervention }\end{array}$ & $\begin{array}{l}100200 \\
\text { Favors co }\end{array}$ \\
\hline
\end{tabular}

Fig. 5. Forest plot of cortisol measurements $\left(\mathrm{nmol} \mathrm{I}^{-1}\right)$ occurring within $60 \mathrm{~min}$ of castration for trials comparing the pain responses of piglets that received a variety of non-steroidal anti-inflammatory to no anti-inflammatory.

assessment of $\beta$-endorphins or norepinephrine in castrated pigs receiving NSAIDS. Individual results for vocalisation outcomes and pain-related behaviours are provided in Tables 6 and 7, respectively.

\section{Synthesis of results}

Prior to attempting quantitative synthesis, but after data extraction, the content experts were asked to determine 


\begin{tabular}{|c|c|c|c|c|c|c|c|c|c|c|}
\hline \multirow{2}{*}{$\begin{array}{l}\text { Study or subgroup } \\
1.2 .1 \text { Meloxicam }\end{array}$} & \multicolumn{2}{|c|}{$\begin{array}{c}\text { Intervention } \\
\end{array}$} & Total & \multicolumn{3}{|c|}{ Control } & \multirow[t]{2}{*}{ Weight } & \multirow[t]{2}{*}{$\begin{array}{c}\text { Mean difference } \\
\text { IV, Random, } 95 \% \mathrm{Cl}\left(\mathrm{nmol} \mathrm{I}^{-1}\right)\end{array}$} & \multicolumn{2}{|c|}{$\begin{array}{c}\text { Mean difference } \\
\text { IV, Random, } 95 \% \mathrm{Cl}\left(\mathrm{nmol} \mathrm{I}{ }^{-1}\right)\end{array}$} \\
\hline & & & & & & & & & & \\
\hline Langhoff (2009) & 38 & 29 & 25 & 73 & 28 & 28 & $66.1 \%$ & $-35.00[-50.39,-19.61]$ & 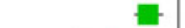 & \\
\hline Tenbergen (2012b) & 79.2 & 44.7 & 25 & 156.4 & 105.4 & 20 & $33.9 \%$ & $-77.20[-126.60,-27.80]$ & $\longrightarrow$ & \\
\hline Wavreille (2012)(\%) & 0 & 0 & 15 & 0 & 0 & 14 & & Not estimable & & \\
\hline Zols (2006) & 60 & 0 & 72 & 110 & 0 & 45 & & Not estimable & & \\
\hline Subtotal $(95 \% \mathrm{Cl})$ & & & 137 & & & 107 & $100.0 \%$ & $-49.30[-88.45,-10.15]$ & & \\
\hline \multicolumn{11}{|c|}{$\begin{array}{l}\text { Heterogeneity. Tau }{ }^{2}=541.91 ; \mathrm{Chi}^{2}=2.55, \mathrm{df}=1(P=0.11) ; I^{2}=61 \% \\
\text { Test for overall effect: } Z=2.47(P=0.01)\end{array}$} \\
\hline \multicolumn{11}{|l|}{ 1.2.2 Tolfenamic acid } \\
\hline $\begin{array}{l}\text { Wavreille }(2012)(\%) \\
\text { Subtotal }(95 \% \mathrm{Cl})\end{array}$ & 0 & 0 & $\begin{array}{l}17 \\
17\end{array}$ & 0 & 0 & $\begin{array}{l}14 \\
14\end{array}$ & & $\begin{array}{l}\text { Not estimable } \\
\text { Not estimable }\end{array}$ & & \\
\hline \multicolumn{11}{|c|}{$\begin{array}{l}\text { Heterogeneity. not applicable } \\
\text { Test for overall effect. not applicable }\end{array}$} \\
\hline \multicolumn{11}{|c|}{ 1.2.3 Flunixin meglumine } \\
\hline Langhoff (2009) & 31 & 37 & 26 & 73 & 28 & 28 & $63.9 \%$ & $-42.00[-59.60,-24.40]$ & & \\
\hline $\begin{array}{l}\text { Sutherland }(2010) \\
\text { Subtotal }(95 \% \mathrm{Cl})\end{array}$ & 150.36 & 107.7 & $\begin{array}{l}10 \\
36\end{array}$ & 126 & 37.63 & $\begin{array}{l}10 \\
38\end{array}$ & $\begin{array}{r}36.1 \% \\
100.0 \%\end{array}$ & $\begin{array}{r}24.36[-46.35,95.07] \\
-\mathbf{1 8 . 0 2}[-\mathbf{8 0 . 5 0}, \mathbf{4 4 . 4 6 ]}\end{array}$ & & \\
\hline \multicolumn{11}{|c|}{$\begin{array}{l}\text { Heterogeneity. Tau }{ }^{2}=1510.73 ; \mathrm{Chi}^{2}=3.19, \mathrm{df}=1(P=0.07) ; I^{2}=69 \% \\
\text { Test for overall effect: } Z=0.57(P=0.57)\end{array}$} \\
\hline \multicolumn{11}{|l|}{ 1.2.4 Ketoprofen } \\
\hline $\begin{array}{l}\text { Tenbergen }(2012 \mathrm{c}) \\
\text { Subtotal }(95 \% \mathrm{Cl})\end{array}$ & 46.1 & 17 & $\begin{array}{l}12 \\
12\end{array}$ & 127.8 & 73.8 & $\begin{array}{l}15 \\
15\end{array}$ & $\begin{array}{l}100.0 \% \\
\mathbf{1 0 0 . 0} \%\end{array}$ & $\begin{array}{l}-81.70[-120.27,-43.13] \\
-81.70[-120.27,-43.13]\end{array}$ & & \\
\hline \multicolumn{11}{|c|}{ Heterogeneity. not applicable } \\
\hline \multicolumn{11}{|l|}{ 1.2.5 Carprofen } \\
\hline $\begin{array}{l}\text { Langhoff }(2009) \\
\text { Subtotal }(95 \% \mathrm{Cl})\end{array}$ & 42 & 27 & $\begin{array}{l}15 \\
15\end{array}$ & 73 & 28 & $\begin{array}{l}28 \\
28\end{array}$ & $\begin{array}{l}100.0 \% \\
\mathbf{1 0 0 . 0} \%\end{array}$ & $\begin{array}{l}-31.00[-48.15,-13.85] \\
-31.00[-48.15,-13.85]\end{array}$ & & \\
\hline \multicolumn{11}{|c|}{$\begin{array}{l}\text { Heterogeneity. not applicable } \\
\text { Test for overall effect: } Z=3.54(P=0.0004)\end{array}$} \\
\hline & & & & & & & & & $\begin{array}{l}\vdash \\
-200-100 \quad 0 \\
\text { Favors intervention }\end{array}$ & $\frac{1}{100}$ \\
\hline
\end{tabular}

Fig. 6. Forest plot of cortisol measurements $\left(\mathrm{nmol} \mathrm{I}^{-1}\right)$ occurring between 1 and $24 \mathrm{~h}$ of castration for trials comparing the pain responses of piglets that received a variety of non-steroidal anti-inflammatory to no anti-inflammatory.

appropriate subgroup analyses. This approach was necessary because at the protocol stage only some but not all interventions in the body of work were known. Because of differing pharmacokinetics within the general anesthesia protocols, the expert group suggested that general anesthesia protocols should always be considered separately. Therefore, it was decided that no summary effect of general anesthetic should be calculated. However, for the NSAIDs and local anesthetic protocols, a priori it was considered reasonable to test the hypothesis that the subgroups were not a source of clinical heterogeneity.

\section{Synthesis of results for castration and general anesthetic protocols}

Only $\mathrm{CO}_{2} / \mathrm{O}_{2}$ anesthesia had more than one study with estimable outcome for cortisol. Fig. 2 illustrates there was considerable statistical heterogeneity across studies for this intervention $\left(\chi^{2}=17.10 ; P=0.002 ; I^{2}=88 \%\right)$; therefore, while the summary effect for cortisol levels under $60 \mathrm{~min}$, that does not favor $\mathrm{CO}_{2} / \mathrm{O}_{2}$ anesthesia (summary mean difference: 33.97; 95\% CI: $\quad-57.41-125.35 ; \quad P=0.47)$. Figure 3 shows that cortisol levels for piglets between 1 and $24 \mathrm{~h}$ post-castration and the effect of $\mathrm{CO}_{2} / \mathrm{O}_{2}$ anesthesia on cortisol seemed favorable (summary mean difference: -59.97 ; 95\% CI: -92.78 to -27.17 ; $P<0.001)$, and there appeared to be little evidence of heterogeneity between these two studies $\left(\chi^{2}=1.10 ; P=0.29 ; I^{2}=9 \%\right)$.
The forest plot in Fig. 4 also provided the results of the meta-analysis of $\beta$-endorphins. Data were available for several protocols, and the subgroup analysis suggested provided no reason to reject the null hypothesis that these protocols can be combined (test for subgroup differences $P=0.91)$. As all studies reported no significant change in $\beta$-endorphins, the conclusion was that general anesthetics did not affect $\beta$-endorphins measured within $60 \mathrm{~min}$ of castration. No synthesis of vocalisation outcomes was conducted for general anesthesia protocols and castration. Furthermore, we did not attempt synthesis of the pain-related behaviors as only one studied reported completely.

\section{Synthesis of results for castration and local anesthetic protocols}

Because the results were incompletely reported, it was not possible to conduct quantitative synthesis of cortisol, $\beta$-endorphins, and norepinephrine for local anesthetic protocols. For the vocalisation outcomes, although we attempted to limit the outcomes to those that had common units ( $\mathrm{Hz}, \mathrm{dB}$, and rates) to increase the opportunity for synthesis even within this restriction, the metric reported by authors was very diverse. For example, some authors reported peak amplitude and others reported call intensity, for some outcomes the expectation was a decrease, for other outcomes the expectation was an increase, and therefore was unclear 
Table 5. List of outcomes extracted and frequency of reporting based on time points and management procedure

\begin{tabular}{|c|c|c|c|c|c|c|c|c|}
\hline \multirow[b]{2}{*}{ Outcome } & \multicolumn{2}{|l|}{ Castration } & \multicolumn{2}{|c|}{ Tail docking } & \multicolumn{2}{|c|}{ Ear notching } & \multicolumn{2}{|c|}{ Teeth clipping } \\
\hline & $0-60 \min$ & $1-24 \mathrm{~h}$ & $0-60 \mathrm{~min}$ & $1-24 \mathrm{~h}$ & $0-60 \mathrm{~min}$ & $1-24 \mathrm{~h}$ & $0-60 \min$ & $1-24 \mathrm{~h}$ \\
\hline ACTH & 5 & 0 & 1 & 0 & 0 & 0 & 0 & 0 \\
\hline$\beta$-endorphins & 6 & 2 & 0 & 0 & 0 & 0 & 0 & 0 \\
\hline Body temperature & 1 & 2 & 0 & 0 & 0 & 0 & 0 & 0 \\
\hline Cortisol & 16 & 13 & 3 & 4 & 0 & 0 & 0 & 0 \\
\hline Epinephrine & 4 & 0 & 0 & 0 & 0 & 0 & 0 & 0 \\
\hline Haptoglobin & 0 & 0 & 0 & 0 & 0 & 0 & 0 & 0 \\
\hline Heart rate & 4 & 0 & 0 & 0 & 0 & 0 & 0 & 0 \\
\hline Norepinephrine & 4 & 0 & 0 & 0 & 0 & 0 & 0 & 0 \\
\hline Respiratory rate & 4 & 0 & 0 & 0 & 0 & 0 & 0 & 0 \\
\hline Substance P & 1 & 1 & 0 & 0 & 0 & 0 & 0 & 0 \\
\hline \multicolumn{9}{|l|}{ Vocalization } \\
\hline Call duration & 3 & 0 & 0 & 0 & 0 & 0 & 0 & 0 \\
\hline Call rate & 2 & 0 & 0 & 0 & 0 & 0 & 0 & 0 \\
\hline Main frequency & 4 & 0 & 1 & 0 & 0 & 0 & 0 & 0 \\
\hline Peak amplitude & 2 & 0 & 0 & 0 & 0 & 0 & 0 & 0 \\
\hline Peak frequency & 3 & 0 & 0 & 0 & 0 & 0 & 0 & 0 \\
\hline \multicolumn{9}{|l|}{ Activity event } \\
\hline Escape attempts & 1 & 0 & 0 & 0 & 0 & 0 & 0 & 0 \\
\hline Activity state & & & & & & & & \\
\hline Lying & 5 & 7 & 3 & 2 & 0 & 0 & 0 & 0 \\
\hline Playing & 3 & 4 & 0 & 0 & 0 & 0 & 0 & 0 \\
\hline Running & 2 & 2 & 0 & 0 & 0 & 0 & 0 & 0 \\
\hline Sitting & 3 & 4 & 2 & 1 & 0 & 0 & 0 & 0 \\
\hline Aggression & 2 & 2 & 0 & 0 & 0 & 0 & 0 & 0 \\
\hline Avoidance & 3 & 3 & 2 & 0 & 0 & 0 & 0 & 0 \\
\hline \multicolumn{9}{|l|}{ Body movement } \\
\hline Head shaking & 0 & 0 & 2 & 0 & 2 & 0 & 2 & 0 \\
\hline Rear end movement & 7 & 3 & 3 & 0 & 2 & 0 & 2 & 0 \\
\hline \multicolumn{9}{|l|}{ Feeding event } \\
\hline Suckling/nursing & 3 & 5 & 0 & 0 & 0 & 0 & 0 & 0 \\
\hline Teat seeking/udder movement & 2 & 3 & 1 & 1 & 0 & 0 & 0 & 0 \\
\hline Teeth champing/chewing & 0 & 0 & 2 & 0 & 2 & 0 & 2 & 0 \\
\hline \multicolumn{9}{|l|}{ Feeding state } \\
\hline Suckling/nursing & 2 & 4 & 0 & 0 & 0 & 0 & 0 & 0 \\
\hline Teat seeking/udder movement & 0 & 1 & 2 & 1 & 0 & 0 & 0 & 0 \\
\hline
\end{tabular}

Outcomes never reported: eeg and ecg, teeth champing/chewing, ear flicking, urination, defecation.

that these should be quantitatively combined. Furthermore, the reporting of measures of variation was poor (see Table 6). Consequently no synthesis was possible to combine these data or quantify of the effect of NSAIDS on vocalisation. In general, the data reported in Table 6 show that the point estimates of vocalisation outcomes were lower in treated groups, as would be expected. However, the magnitude of effect cannot be determined. Many studies that did report these outcomes also reported blinding of outcome assessment as shown in Table 5. For the pain-related behaviors, again reporting was incomplete, precluding quantitative synthesis. Even making inference about point estimates was not even.

Synthesis of results for castration and NSAID protocols Although all three NSAIDS (meloxicam, ketoprofen, and flunixin meglumine) showed reductions in cortisol levels in the treated animals, an assessment across the NSAIDS subgroups suggested considerable heterogeneity. Therefore, an across protocol summary effect measure was not calculated $\left(I^{2}=94.4 \% ; \quad \chi^{2}=53.33\right.$; $P=0.00001$ ) (Fig. 5). Therefore, the results are discussed separately.

Figure 5 provides the results of the meta-analysis of the protocols that assessed the impact of use of NSAIDs on blood cortisol levels under $60 \mathrm{~min}$ for piglets that received meloxicam, ketoprofen, or flunixin meglumine. The difference in magnitude reported by the three studies that assessed meloxicam is reflected in the results of the tests for heterogeneity among these studies $\left(\chi^{2}=6.08\right.$; $\left.P=0.05 ; I^{2}=67 \%\right)$. With only three studies it was not possible to explore sources of heterogeneity. Therefore, while the summary effect measure does support the notion that meloxicam does reduce cortisol measured within $60 \mathrm{~min}$ of the procedure (MD: $-92.02 ; 95 \% \mathrm{CI}$ : -158.12 to $-25.92 ; \quad P=0.006)$, the width of the 
Table 6. Results from individual studies for vocalisation outcomes for piglets undergoing castration (no tail-docking studies reported vocalisation)

\begin{tabular}{|c|c|c|c|c|c|c|c|}
\hline Study & Author's description of outcome & Units & Intervention & $\begin{array}{l}\text { Intervention group result } \\
\text { (mean }(\mathrm{SD}) \text { or rate }(\mathrm{SD}) \\
\text { or proportion }(\mathrm{SD}))\end{array}$ & $\begin{array}{l}\text { Number in } \\
\text { intervention } \\
\text { group }\end{array}$ & $\begin{array}{l}\text { Placebo group result } \\
\text { (mean }(\mathrm{SD}) \text { or rate }(\mathrm{SD}) \\
\text { or proportion }(\mathrm{SD}))\end{array}$ & $\begin{array}{l}\text { Number in } \\
\text { comparison } \\
\text { group }\end{array}$ \\
\hline \multicolumn{8}{|c|}{ General anesthesia } \\
\hline $\begin{array}{l}\text { Rault and } \\
\text { Lay (2011) }\end{array}$ & $\begin{array}{l}\text { Vocalisation (both high frequency (i.e. } \\
\text { squeals) and low frequency (i.e. grunts)) } \\
\text { length during castration }\end{array}$ & $\%$ & $\mathrm{~N}_{2} \mathrm{O} / \mathrm{O}_{2}$ & $81.45(21.61)$ & 12 & $79.93(21.61)$ & 12 \\
\hline \multicolumn{8}{|c|}{ Local anesthesia } \\
\hline $\begin{array}{l}\text { Courboulay } \\
\text { et al. (2010) }\end{array}$ & Average intensity of cries & $\mathrm{dB}$ & Lidocaine & $103.4(11.3)$ & NR & $113(4)$ & NR \\
\hline $\begin{array}{l}\text { Hansson } \\
\text { et al. (2011) }\end{array}$ & Call intensity & $\mathrm{dB}$ & Lidocaine & $103(11)$ & 139 & $11.9(8)$ & 139 \\
\hline $\begin{array}{l}\text { Horn et al. } \\
\text { (1999) }\end{array}$ & Grunts & $\begin{array}{l}\text { Number of } \\
\text { grunts }\end{array}$ & Lidocaine & 38.7 (NR) & 12 & $45.1(\mathrm{NR})$ & 12 \\
\hline $\begin{array}{l}\text { Horn et al. } \\
\text { (1999) }\end{array}$ & Cries & $\begin{array}{l}\text { Number of } \\
\text { cries }\end{array}$ & Lidocaine & $112.2(\mathrm{NR})$ & 12 & $132.4(\mathrm{NR})$ & 12 \\
\hline $\begin{array}{l}\text { Horn et al. } \\
(1999)\end{array}$ & Grunts & $\begin{array}{l}\text { Number of } \\
\text { grunts }\end{array}$ & Lidocaine & 36.6 (NR) & 12 & $45.1(\mathrm{NR})$ & 12 \\
\hline $\begin{array}{l}\text { Horn et al. } \\
(1999)\end{array}$ & Cries & $\begin{array}{l}\text { Number of } \\
\text { cries }\end{array}$ & Lidocaine & $148.8(\mathrm{NR})$ & 12 & $132.4(\mathrm{NR})$ & 12 \\
\hline $\begin{array}{l}\text { Kluivers- } \\
\text { Poodt et al. } \\
\text { (2012) }\end{array}$ & $\begin{array}{l}\text { Main frequency (frequency of highest } \\
\text { amplitude in the mean spectra of the } \\
\text { call) }\end{array}$ & $\mathrm{Hz}$ & Lidocaine & $3894(409)$ & 32 & $4464(289)$ & 32 \\
\hline $\begin{array}{l}\text { Kluivers- } \\
\text { Poodt et al. } \\
(2012)\end{array}$ & Call rate & $\begin{array}{l}\text { Number of } \\
\text { calls per } \\
\text { seconds }\end{array}$ & Lidocaine & $1.052(0.59)$ & 32 & $1.111(0.48)$ & 32 \\
\hline $\begin{array}{l}\text { Kluivers- } \\
\text { Poodt et al. } \\
\text { (2012) }\end{array}$ & $\begin{array}{l}\text { Peak amplitude (maximum amplitude of } \\
\text { a call) }\end{array}$ & $\mathrm{dB}$ & Lidocaine & $-33.5(12.44)$ & 32 & $-25.2(7.63)$ & 32 \\
\hline $\begin{array}{l}\text { Marx et al. } \\
(2003)\end{array}$ & Call energy & $\mathrm{dB}$ & Lidocaine & 89.6 (NR) & $N R$ & $90.2(\mathrm{NR})$ & NR \\
\hline $\begin{array}{l}\text { Marx et al. } \\
(2003)\end{array}$ & Grunts/squeals/screams & $\begin{array}{l}\text { Number of } \\
\text { calls per } \\
\text { animal }\end{array}$ & Lidocaine & 73.25 (NR) & NR & 84.94 (NR) & NR \\
\hline $\begin{array}{l}\text { Marx et al. } \\
(2003)\end{array}$ & Main frequency & $\mathrm{Hz}$ & Lidocaine & $4430(\mathrm{NR})$ & NR & 3943 (NR) & NR \\
\hline $\begin{array}{l}\text { Rittershaus } \\
\text { et al. (2009) }\end{array}$ & Scream frequency & $\begin{array}{l}\text { Increase in } \\
\text { sounds per } \\
\text { second }\end{array}$ & $\begin{array}{l}\text { Chlorethyl } \\
\text { cooling } \\
\text { spray }\end{array}$ & $0.4(\mathrm{NR})$ & NR & $0.7(\mathrm{NR})$ & NR \\
\hline $\begin{array}{l}\text { White et al. } \\
\text { (1995) }\end{array}$ & $\begin{array}{l}\text { Mean frequency with the highest energy } \\
\text { (HEF) }\end{array}$ & $\mathrm{Hz}$ & Lidocaine & $1300(\mathrm{NR})$ & 86 & 3100 (NR) & 86 \\
\hline \multicolumn{8}{|c|}{ Non-steroidal anti-inflammatory drug (NSAID) } \\
\hline $\begin{array}{l}\text { Courboulay } \\
\text { et al. (2010) }\end{array}$ & Average intensity of cries & $\mathrm{dB}$ & Ketoprofen & $113(4)$ & NR & $113(4)$ & NR \\
\hline $\begin{array}{l}\text { Hansson } \\
\text { et al. (2011) }\end{array}$ & Call intensity & $\mathrm{dB}$ & Meloxicam & $111(8)$ & 139 & $111.9(8)$ & 139 \\
\hline
\end{tabular}


confidence interval of the summary effect provides good evidence of the uncertainty about the magnitude of the reduction.

There was no statistical evidence of heterogeneity $\left(\chi^{2}=0.55 ; P=0.46 ; I^{2}=0 \%\right)$ among the studies that assessed the efficacy of ketoprofen based upon blood cortisol levels within 60 min of castration (Schwab et al., 2012; Tenbergen, 2012). The results favored the use of ketoprofen (MD: -235.07 ; 95\% CI: -278.50 to -191.63 ; $P<0.001)$ compared to no intervention.

The data from the four groups (from three studies) that assessed flunixin meglumine (Langhoff et al., 2009; Reiner et al., 2012; Sutherland et al., 2012) favor the use of flunixin meglumine compared with no intervention (MD: -78.44; 95\% CI: -105.98 to $-50.90 ; P<0.001$ ), based on blood cortisol levels under $60 \mathrm{~min}$. There was little evidence of heterogeneity among these studies $\left(\chi^{2}=0.63 ; P=0.89 ; I^{2}=0 \%\right)$.

Figure 6 is a forest plot summarizing the comparisons of blood cortisol levels between 1 and $24 \mathrm{~h}$ for piglets that received NSAIDs. Generally, the observations were favorable, suggesting that these NSAIDS were consistently associated with reductions in cortisol. The assessment of heterogeneity across subgroups suggested the effect was consistent across all the protocols $\left(I^{2}=51.9 \% ; \chi^{2}\right.$ for subgroup differences $=6.24 ; P=0.10$ ).

\section{Assessment of risk of bias across studies}

Formal statistical assessment of publication bias or selective reporting bias was not feasible due to limited availability of data for each intervention-outcome-procedure combination.

\section{Discussion}

\section{Summary of evidence}

Pain mitigation in piglets between one and 28 days of age, undergoing castration, tail docking, ear notching, or teeth clipping will likely become a higher priority area for consumers and retailers as attention moves beyond gestation sow housing. There are currently no US Food and Drug Administration (FDA) approved drugs for pain mitigation in piglets destined for human consumption. Furthermore, the mechanism of pain mitigation by available drugs is poorly understood in swine and is extrapolated from other scientific evidence. Although a reasonably large body of work was available for evaluation, a number of issues reduced our ability to confidently assess the comparative efficacy of general anesthetic, local anesthetic, or NSAID protocols. Although interesting, the exact relationship between the outcomes reported and pain is not within the scope of this review. Instead, we summarized the associations between the 
Table 7. Extracted pain-related behavioral outcomes for castration and tail docking

\begin{tabular}{|c|c|c|c|c|c|c|c|}
\hline Study & Author's description of outcome & Units & Intervention & $\begin{array}{l}\text { Intervention group } \\
\text { result (mean (SD) } \\
\text { or rate }(\mathrm{SD}) \text { or } \\
\text { proportion }(\mathrm{SD}))\end{array}$ & $\begin{array}{l}\text { Active group } \\
n\end{array}$ & $\begin{array}{l}\text { Placebo group } \\
\text { result (mean }(\mathrm{SD}) \\
\text { or rate }(\mathrm{SD}) \text { or } \\
\text { proportion }(\mathrm{SD}))\end{array}$ & $\begin{array}{l}\text { Placebo } \\
\text { group } n\end{array}$ \\
\hline \multicolumn{8}{|c|}{ General anesthesia within $60 \mathrm{~min}$} \\
\hline Kohler et al. (1998) & Pain reaction score & Score & $\mathrm{CO}_{2} / \mathrm{O}_{2}$ & $0(\mathrm{~N} / \mathrm{A})$ & 10 & $9(\mathrm{~N} / \mathrm{A})$ & 10 \\
\hline Kohler et al. (1998) & Pain reaction score & Score & $\begin{array}{l}\text { Halothane/ } \\
\mathrm{O}_{2}\end{array}$ & $5(\mathrm{~N} / \mathrm{A})$ & 10 & $9(\mathrm{~N} / \mathrm{A})$ & 10 \\
\hline Rault and Lay (2011) & Tail wag & $\begin{array}{l}\text { Average of } 24 \\
\text { scans }\end{array}$ & $\mathrm{N}_{2} \mathrm{O} / \mathrm{O}_{2}$ & $1.73(0.9)$ & 12 & $2.46(3.11)$ & 12 \\
\hline \multicolumn{8}{|c|}{ Local anesthesia within 60 min of procedure } \\
\hline $\begin{array}{l}\text { Courboulay et al. } \\
(2010)\end{array}$ & $\begin{array}{l}\text { Frequency of movement of the hind } \\
\text { quarters }\end{array}$ & $\%$ of piglets & Lidocaine & $87.5(\mathrm{NR})$ & NR & $N R(N R)$ & NR \\
\hline Horn et al. (1999) & Duration of defensive movement & Seconds & Procaine & $32(12.7825)$ & 12 & $44.98(12.78)$ & 12 \\
\hline Leidig et al. (2009) & Intensity of defense behavior (scale $0-4$ ) & $\%$ & Lidocaine & $\begin{array}{l}\text { Reported as } \\
\text { medians } \\
\text { and quartiles }\end{array}$ & NR & $\begin{array}{l}\text { Reported as } \\
\text { medians } \\
\text { and quartiles }\end{array}$ & NR \\
\hline \multicolumn{8}{|c|}{ Local anesthesia $1-24 \mathrm{~h}$ post-procedure } \\
\hline $\begin{array}{l}\text { Courboulay et al. } \\
\text { (2010) }\end{array}$ & Tail wagging & $\%$ of views & Lidocaine & $3.5(4.3)$ & NR & NR (NR) & NR \\
\hline \multicolumn{8}{|c|}{ NSAID within 60 min post-procedure } \\
\hline $\begin{array}{l}\text { Courboulay et al. } \\
(2010)\end{array}$ & Tail wagging & & Ketoprofen & $0.9(2.4)$ & & $1.1(3)$ & \\
\hline Schwab et al. (2012) & Mean behavior score at $1 \mathrm{~h}$ & & Ketoprofen & 0.7 (NR) & 50 & $1.1(\mathrm{NR})$ & 50 \\
\hline $\begin{array}{l}\text { NSAID } 1-24 \text { h post-pr } \\
\text { Courboulay et al. } \\
\text { (2010) }\end{array}$ & $\begin{array}{l}\text { Tedure } \\
\text { Tail wagging }\end{array}$ & $\%$ of views & Ketoprofen & $1.6(2.1)$ & NR & $8.6(11)$ & \\
\hline Reiner et al. (2012) & Duration of flinching tail & Seconds & Flunixin & $55.3(82.15)$ & 30 & $33.5(41.62)$ & 30 \\
\hline Reiner et al. (2012) & Duration of flinching tail & Seconds & Flunixin & $42.9(65.72)$ & 30 & $33.5(41.62)$ & 30 \\
\hline Reiner et al. (2012) & Duration of flinching tail & Seconds & Meloxicam & $54.7(62.44)$ & 30 & $33.5(41.62)$ & 30 \\
\hline Schwab et al. (2012) & Mean behavior score at $2 \mathrm{~h}$ & Score & Ketoprofen & $0.8(\mathrm{NR})$ & 50 & $1.1(\mathrm{NR})$ & 50 \\
\hline $\begin{array}{l}\text { General anesthesia, ta } \\
\text { Sutherland et al. } \\
\text { (2012) } \\
\text { General anesthesia, ta }\end{array}$ & $\begin{array}{l}\text { docking within } 60 \text { min post-procedure } \\
\% \text { of time spent performing behavior } \\
\text { docking } 1-24 \mathrm{~h} \text { post-procedure }\end{array}$ & $\begin{array}{l}\text { Pain-like } \\
\text { behaviors }\end{array}$ & $100 \% \mathrm{CO}_{2}$ & $4.14(0.34)$ & 10 & $2.11(1.42)$ & 10 \\
\hline $\begin{array}{l}\text { Sutherland et al. } \\
\text { (2012) }\end{array}$ & $\%$ of time spent performing behavior & $\begin{array}{l}\text { Pain-like } \\
\text { behaviors }\end{array}$ & $100 \% \mathrm{CO}_{2}$ & $0.04(1.48)$ & 10 & $0.86(0.34)$ & 10 \\
\hline
\end{tabular}

NR, not reported. 
outcomes measured and the interventions, and the end user must make use of that to make inferences about pain. The end user may then determine if the association is sufficient to warrant use because adoption of a product must be based on: (1) the expected association between the outcome and pain, (2) the magnitude of the effect, (3) values and preferences, (4) balance of benefits and harms based on other outcomes, and (5) resources. For our project, that step was a separate part and is reported in the companion paper.

Using the data in Figs. 2 and 3, the available evidence suggests general anesthesia protocols did not reduce cortisol levels under $60 \mathrm{~min}$. For the two studies that assessed cortisol from 1 to $24 \mathrm{~h}$ using $\mathrm{CO}_{2} / \mathrm{O}_{2}$ anesthesia, the summary mean difference was lower in animals that received the $\mathrm{CO}_{2} / \mathrm{O}_{2}$ anesthesia. The study that reported the significant effect also did not report random allocation to group, whereas the study with the confidence interval that included the null value did report random allocation to group. The potential for selection bias to affect the outcome is therefore unclear. Both studies failed to report blinding however the potential for bias is likely low. End users would have to determine if such evidence is sufficient to warrant use when compared with other outcomes including the potential for adverse events when general anesthesia is employed.

From Fig. 4, the data also suggest that $\mathrm{CO}_{2} / \mathrm{O}_{2}$ anesthesia is associated with lower mean of $\beta$-endorphins when measured within $60 \mathrm{~min}$ of castration. The association between of $\beta$-endorphins and pain in piglets is unclear; therefore, end users would have to determine if such evidence is sufficient to warrant use.

For the NSAIDs, meloxicam, and ketoprofen, the summary mean difference for cortisol measure consistently lower in animals receiving these products under $60 \mathrm{~min}$ and 1 to $24 \mathrm{~h}$. For flunixin meglumine, the summary measure was calculated to not include the null value when cortisol was measured under $60 \mathrm{~min}$. The potential for selection to affect these outcomes is unclear, as the studies contain a mixture of studies that reported randomization or failed to report randomization (Table 3). Similarly, blinding was inconsistently reported however the potential for misclassification bias for this outcome is likely low.

Although authors reported vocalisation and painrelated behavior measures, quantitative synthesis approaches were not suited for evaluating these outcomes. Methods described were neither validated nor was there harmonization among researchers. One option would be to standardize these outcomes using standardized mean difference, but this was not considered suitable because the expected direction of benefit (i.e. should the intervention increase or decrease the outcome if effective) was not clear from the original manuscript. Therefore, we simply summarized the extracted vocalisation and pain-related measures and presented them in Tables 6 and 7 . In these tables, it is possible to see that often the point estimate is lower in the treatment group; however, it is also possible to see that measures of variation are often not reported. Consequently, it seems inappropriate to draw conferences from these point estimates. Depending upon how the outcome is measured, the potential for bias could be high. The end user can identify a study of interest and determine the use of blinding by referring to Table 3 .

Meaningful heterogeneity was a common feature of the meta-analysis. Although the opportunity to explore source of heterogeneity was not available due to limited data, a major source of heterogeneity could be the methodological approaches used by individual investigators. Some of these sources might include when procedures were administered. For example, NSAIDs were administered at variable times prior to castration, e.g. $15 \mathrm{~min}, 30 \mathrm{~min}$ or $1 \mathrm{~h}$ before the procedure. There were also differences in when outcomes were assessed, e.g. cortisol assessed at $90 \mathrm{~min}, 2 \mathrm{~h}$, or $4 \mathrm{~h}$ after castration. There was also failure by authors to report key components of the study design such as blinding and the approach to allocation to groups.

\section{Limitations}

\section{Review-level limitations}

The review was conducted based on the approaches recommended by standard groups associated with systematic reviews with little deviation from the protocol. One outstanding aspect of the review was the translation of studies to be included, a feature that is often not included in reviews. With respect to the review methodology, two usually desirable aspects of systematic reviews that include a meta-analysis are missing: an assessment of publication bias and a quantitative assessment of sources of (rather than just the presence of) heterogeneity using meta-regression or similar methodologies. Both of these features are missing because of insufficient data to conduct appropriate analyses rather than simple omission.

Although there may be concern about the funding agency and the extent to which the experts in the project were associated with pharmaceutical products, we propose that the risk of bias was controlled by several factors. First, the experts included were chosen based on the relevance of their expertise to the review question. This expertise that made them outstanding contributors to the review process is the same factor that encourages interaction with companies exploring pain-mitigation products. Individuals with fewer connections may have fewer conflicts of interest but also be less knowledgeable. This in turn could have resulted in less skill and knowledge to prepare the comprehensive review protocol that required little modification after being adopted a key tool for preventing bias in the systematic review. As a safeguard against bias, the protocol was also submitted 
for external review to welfare and pain management experts in North America, Europe, and Australasia. Furthermore, the review team responsible for screening for relevance and extracting the data were not involved in welfare research prior to this review and are not affiliated with companies that produce pain-mitigation products. These extracted data were submitted for external validation to welfare and pain management experts in North America, Europe, and Australasia. Duplicate processes were used where possible and the search for relevant studies was, in our opinion, extensive.

\section{Study-level limitations}

The following limitations that prevented us from fully achieving our goals: (1) the scope of procedures studies and (2) the comprehensiveness of reporting of study features.

\section{The scope of procedures studied}

There was a lack of studies that assess the efficacy of pain mitigation strategies on castration, tail docking, ear notching, and teeth clipping. The data in Table 3 show that the later three procedures are rarely the subject of published studies.

\section{The approach to reporting study features}

The approach to reporting design features that would enable us to assess bias further prevented us for some topics drawing any conclusions, as the uncertainty was so great. Knowledge of bias is important for interpretation of the results of studies. The design features we wanted to assess were approaches to reducing selection bias with randomization to group. We also wanted to assess approaches to reducing differential misinformation bias. Differential misinformation bias can occur in all outcomes, even physiological outcomes such as blood cortisol, so the assessment of all outcomes should be blinded. It is the potential for mismeasurement that does differ between outcomes. Frequently, outcomes obtained by human measurement are considered to have a greater potential for measurement bias. For example, potential measurement bias occurs when heart rate is counted via stethoscope compared to heart rate measured by electrocardiography (ECG), or when vocalisations are counted by observers compared with sound-recording equipment because of the potential for human error. A promising pain-related behavioral outcome for castration may be rear end movements, such as easing of the quarters and tail wagging. However, although this outcome is quantifiable, like any diagnostic test, the sensitivity and specificity may be imperfect. If sensitivity and specificity differ across the treatment groups, then the observed effect may differ from the true effect. For this reason, it is critical that researchers report all efforts taken to ensure that non-differential mismeasurement of the outcome does not occur. For example, it is common to report that behavioral data was collected by trained observers, but details about how the observers were trained and measures of intra- and inter-observer reliability are rarely reported.

\section{Outcome-level limitations}

\section{The outcome reported}

Many outcomes that our experts considered important enough to request be extracted were frequently not reported, and these suggest gaps in the knowledge base (Table 5). Relevant to the review, an ideal outcome variable should be either present or elevated when piglets receive a painful procedure and either remain unchanged or be absent when they do not. There was also an assumption that these pain outcomes would be reduced or absent if a procedure is performed when analgesia or anesthesia is provided. There can be confounding between responses to stressful and painful events in terms of some aspects of behavior, but this issue is particularly problematic for physiological and biochemical parameters, such as cortisol or heart rate, that are associated with general stress responses and/or inflammatory responses. Experimental designs involving cognitive responses, such as preference or avoidance testing, provide opportunity to empirically 'ask' animals about their pain perception versus biological responses (Danbury et al., 2000), but these have not been used to explore pain in piglets. In the absence of fundamental science informing us about the sensitivity and specificity of neurophysiological, endocrine, or behavioral outcomes associated with pain, we are restricted in our ability to compile a reliable database of the efficacy and reliability of pain-mitigating interventions.

Furthermore, the approach to reporting outcomes often limited our ability to summarize the data. For many outcomes there was incomplete reporting of important summary measures (i.e. mean, SD, or SE). An example of a major impact of this was for the intervention local anesthesia. Although numerous studies reported numerous outcomes reported this intervention, the only outcome where data from multiple studies could be extracted was vocalisations. However, from the 14 studies that reported vocalisations for local anesthetics, it was only possible to extract measures of variation from five (36\%) (Table 6). Even within these studies it was not possible to quantitatively combine the data because of the different outcomes used. For example, Courboulay et al. (2010) used a sonometer to record the 'intensity of cries,' whereas Cordeiro et al. (2012) used a microphone with digital software to record the maximum amplitude of a call. Both outcomes shared the same units (dB); however, the latter was reported as a negative value. Rittershaus et al. (2009) reported an increase in sounds per second, while Kluivers-Poodt et al. (2012) reported the number of calls per second. Therefore, even when authors reported measures, quantitative synthesis approaches were not suited for evaluating outcomes with such heterogeneity. 


\section{Conclusions}

Given the above caveats, we reached the following conclusions using the 52 studies from our evidence synthesis. $\mathrm{CO}_{2} / \mathrm{O}_{2}$ anesthesia and the NSAIDs meloxicam, ketoprofen, and flunixin meglumine may be useful in mitigating pain 1 to $24 \mathrm{~h}$ after castration based on cortisol levels. It was not possible, using our approach to research synthesis, to reach a conclusion about the magnitude of the efficacy of local anesthetics or other approaches to general anesthesia. Our conclusion about $\mathrm{CO}_{2} / \mathrm{O}_{2}$ anesthesia and the NSAIDs meloxicam, ketoprofen, and flunixin are based on a handful of studies with design and reporting insufficiencies that could potentially indicate confounding and misclassification bias. Substantial opportunities exist to improve research in the area of pain mitigation in the swine neonate undergoing routine procedures in the areas of study design, the use of validated equipment and assays, and descriptive statistics. For pain-related behavioral outcomes, we suggest using complete ethograms that describe the behaviors/motor patterns, details of how individual pigs are identified, the sampling procedures over time (e.g. scans or continuous), type of equipment used for behavioral observation (live versus digital), and descriptive information such as states or events. For vocalisations outcomes we propose improved reporting of measures of variation and information about the a priori hypothesized direction of effect. These improved approaches should be utilized when performing research and evaluating outcomes across interventions.

\section{Funding}

This project was funded by the National Pork Board (NPB) (grant no. 12-186).

\section{Declaration of interest}

Dr Dzikamunhenga was supported as a postdoctoral fellowship funded by the National Pork Board. Dr O'Connor serves on the Food Safety Committee for the $\mathrm{NPB}$ and receives complimentary travel and lodging to attend meetings. Dr O'Connor has received funding for food safety research from NPB and the current project related to animal welfare. Dr O'Connor has been funded for projects by Pfizer Animal Health unrelated to pain interventions or swine welfare. Dr O'Connor teaches the clinical trial design for swine producers for BoehringerIngelheim Vetmedica. Dr O'Connor is a collaborator on pain-related research funded by Agriculture and Food Research Initiative competitive grants from the USDA National Institute of Food and Agriculture (grant no. 201267021-19363). Ms Gould was partially supported by the
National Pork Board. Dr Coetzee has been a consultant for Intervet-Schering Plough Animal Health (Now Merck), Boehringer-Ingelheim Vetmedica, Zoetis Animal Health, and Norbrook Laboratories Ltd. He has also addressed the Staff College at the US Food and Drug Administration (FDA) Center for Veterinary Medicine (CVM) and has received funding from USDA-CSREES, Animal Protection (Animal Well-being), NRI Grant no. 2008-35204-19238, 2009-65120-05729, no. 2012-67021-19363, and no. 201167021-30369). Dr Johnson serves on the Animal Welfare Committee for the NPB, and the Animal Well-being Committee for IPPA, and receives complimentary travel and lodging to attend meetings. She currently consults for Bob Evans, Elanco, McDonalds, and Murphy-Brown, LLC. In the past, she has consulted for Kroger's. Funding has been received through the following pharmacology agencies: Boehringer-Ingelheim Vetmedica, Elanco, and Pfizer. Dr Johnson has received funding for welfarerelated research from NPB. Dr Johnson has pain-related research that has been funded by Agriculture and Food Research Initiative competitive grants from the USDA National Institute of Food and Agriculture (grant no. 201167021-30369). Dr Locke Karriker has been a consultant for Boehringer-Ingelheim Vetmedica and Bayer Animal Health. He currently has active research funding from the National Pork Board, the Iowa Pork Producers Association, the USDA Higher Education Challenge Competitive Grants Program, and Boehringer-Ingelheim Vetmedica. He is the Director of the Swine Medicine Education Center at Iowa State University. Dr Karriker has pain-related research that has been funded by Agriculture and Food Research Initiative competitive grants from the USDA National Institute of Food and Agriculture (grant no. 2011-67021-30369). Dr Millman currently serves on the Animal Well-being Committee for Iowa Pork Producers Association, and advises McDonalds Corporation, Humane Farm Animal Care, and HyLine International (poultry) on animal welfare issues. Her pain-related research has been funded by Agriculture and Food Research Initiative competitive grants from the USDA National Institute of Food and Agriculture (grant numbers 2012-67021-19363 and 2011-67021-30369), Natural Sciences and Engineering Research Council of Canada, Canadian Foundation for Innovation, National Pork Board, Iowa Pork Producers, Ontario Pork, BoehringerIngelheim Vetmedica (bovine only), Novartis Animal Health (bovine), Intervet-Schering Plough Animal Health (bovine), Pfizer Animal Health (bovine), and MerckMerial (bovine). She has received complimentary travel and lodging to attend meetings from Boehringer-Ingelheim Vetmedica, Inc. Dr McKean serves on the NPB Animal Welfare Committee and has served on the IPPA Swine Health and Welfare Committee and as such received complimentary travel and lodging to attend meetings. McKean has received research grants from the NPB. He does not solicit animal welfare funding or consultation arrangements. Dr McKean has received 
funding for food safety research from NPB and the current project related to animal welfare.

Ms Sherrie Niekamp is employed by the National Pork Board as the Director of Swine Welfare. The National Pork Board has responsibility for Checkoff-funded research, promotion, and consumer information projects and for communicating with pork producers and the public. Through a legislative national Pork Checkoff, pork producers invest US $\$ 0.40$ for each US $\$ 100$ value of hogs sold. Importers of pork products contribute a like amount, based on a formula. The Pork Checkoff funds international, national, and state programs in advertising and promotion, consumer information, and research in production improvement, swine health, pork safety, public health, animal welfare, and environmental management.

\section{Acknowledgment}

Thank you to Dr Andrea Dinkleman for guidance in designing the search.

\section{References}

Beirendonck SV, Driessen B, Verbeke G and Geers R (2011). Behavior of piglets after castration with or without carbon dioxide anesthesia. Journal of Animal Science 89: 33103317.

Bekelman JE and Yahalom J (2009). Quality of radiotherapy reporting in randomized controlled trials of Hodgkin's lymphoma and non-Hodgkin's lymphoma: a systematic review. International Journal of Radiation Oncology, Biology, Physics 73: 492-498.

Brace S, Taylor D and O'Connor AM (2010). The quality of reporting and publication status of vaccines trials presented at veterinary conferences from 1988 to 2003. Vaccine 28: 5306-5314.

Cordeiro AFD, Naas ID, Oliveira SRD, Violaro F and De Almeida ACM (2012). Efficiency of distinct data mining algorithms for classifying stress level in piglets from their vocalization. Engenharia Agricola 32: 208-216.

Courboulay V, Hemonic A, Gadonna M and Prunier A (2010). Effect of local anesthesia or anti-inflammatory treatment on pain associated with piglet castration and on labour demand. Journees de la Recherche Porcine en France 42 : $27-34$.

Danbury TC, Weeks CA, Chambers JP, Waterman-Pearson AE and Kestin SC (2000). Self-selection of the analgesic drug carprofen by lame broiler chickens. Veterinary Record 146: 307-311.

EFSA (2010). Application of systematic review methodology to food and feed safety assessments to support decision making. EFSA Journal 8: Article 1637.

Hansson M, Lundeheim N, Nyman G and Johansson G (2011). Effect of local anaesthesia and/or analgesia on pain responses induced by piglet castration. Acta Veterinaria Scandinavica 53: 34.

Higgins JPT and Green S (eds) (2011). Cochrane Handbook for Systematic Reviews of Interventions Version 5.0.2 [updated September 2009]. The Cochrane Collaboration, 2009. Available from www.cochrane-handbook.org.

Horn T, Marx G and Borell E (1999). Behaviour of piglets during castration with or without local anaesthesia. Deutsche Tierarztliche Wochenschrift 106: 271-274.
Jaggin N, Kohler I, Blum J and Schatzmann U (2001). Castration of newborn piglets under inhalation anesthesia with halothane. Praktische Tierarzt 82: 1054

Kluivers-Poodt M, Houx BB, Robben SR, Koop G, Lambooij E and Hellebrekers LJ (2012). Effects of a local anaesthetic and NSAID in castration of piglets, on the acute pain responses, growth and mortality. Animal 6: 1469-1475.

Kohler I, Moens Y, Busato A, Blum J and Schatzmann U (1998). Inhalation anaesthesia for the castration of piglets: $\mathrm{CO}_{2}$ compared to halothane. Zentralblatt für Veterinärmedizin. Reibe A 45: 625-633.

Lahrmann KH, Kmiec M and Stecher R (2006). Piglet castration with ketamine/azaperone-anaesthesia: concurring with animal welfare, practical, but economic? Prakitsche Tierarzt-Hannover 87: 802-809.

Langhoff R, Zoels S, Barz A, Palzer A, Ritzmann M and Heinritzi K (2009). Investigation about the use of analgesics for the reduction of castration-induced pain in suckling piglets. Berliner Munchener Tierarztliche Wochenschrift 122: 325332.

Lauer S, Zanella A, Kortel A, Henke J, Scharvogel S, Unshelm J, Goldberg M, Eichinger H, Petrowicz O, Brill T and Erhardt W (1994). The $\mathrm{CO}_{2} / \mathrm{O}_{2}$-anaesthesia for castration of male piglets (preliminary results). Deutsche Tierarztliche Wochenschrift 101: 110-113.

Leidig MS, Schumann A, Reiner G, Hertrampf B and Failing K (2009). Pain and discomfort in male piglets during surgical castration with and without local anaesthesia as determined by vocalisation and defence behaviour. Applied Animal Behaviour Science 116: 174-178.

Martin P and Bateson P (2007). Measuring Behaviour: an Introductory Guide. New York, NY, USA: Cambridge University Press.

Marx G, Horn T, Thielebein J, Knubel B and Borell EV (2003). Analysis of pain-related vocalization in young pigs. Journal of Sound and Vibration 266: 687-698.

Mauch C and Bilkei G (2004). Castration of suckling piglets under general anaesthesia - Introduction. Wiener Tierarztliche Monatsschrift 91: 93-98.

Mcglone JJ, Seyfert RD, Fowler S, Kelley KL, Stansbury WF and Hellman JM (1987). Local and general anesthetic effects on behavior and performance of 14-d old castrated and noncastrated piglets. Journal of Animal Science $\mathbf{6 5}$ 231.

Muhlbauer IC, Otten W, Lupping W, Palzer A, Zols S, Elicker S, Ritzmann M and Heinritzi K (2009). Investigation about $\mathrm{CO}_{2}$ gas anaesthesia as an alternative option for piglet castration without anaesthesia. Praklischer Tierarzt 90 460-464.

Nyborg PY, Sorig A, Lykkegaard K and Svendsen O (2000). Nociception after castration of juvenile pigs determined by quantitative estimation of c-Fos expressing neurons in the spinal cord dorsal horn. Dansk Veterinartidsskrift $\mathbf{8 3}$ $16-17$.

Oxman A, Fretheim A, Schunemann H and Sure (2006). Improving the use of research evidence in guideline development: introduction. Health Research Policy and Systems 4: 12.

Prunier A, Bataille G, Meunier-Salaun MC, Bregeon A and Rugraff Y (2001). Influence of tail docking, with or without a cold analgesic spray, on the behaviour, performance and physiology of piglets. Journees de la Recherche Porcine en France 33: 313-318.

Rand JS, Ainscow J, Blackshaw JK, Noonan GJ and Priest J (2002). Oral administration of a $12 \%$ sucrose solution did not decrease behavioural indicators of distress in piglets undergoing tail docking, teeth clipping and ear notching. Animal Welfare 11: 395-404. 
Rault JL and Lay DC (2011). Nitrous oxide by itself is insufficient to relieve pain due to castration in piglets. Journal of Animal Science 89: 3318-3325.

Reiner G, Schollasch F, Hillen S, Willems H, Piechotta M and Failing K (2012). Effects of meloxicam and flunixin on pain, stress and discomfort in male piglets during and after surgical castration. Berl Munch Tierarztl Wochenschr 125: 305-314.

RevMan (2012) Program Review Manager (RevMan) [Computer program] [Computer program]. Available: URL [AccessedAccess Date].

Rittershaus D, Kietzmann M, Schoen PC, Duepjan S and Waldmann KH (2009). Topical anaesthetic techniques during castration of male suckling piglets. In: Sustainable animal husbandry: prevention is better than cure, Volume 1. Proceedings of the 14th International Congress of the International Society for Animal Hygiene, pp. 411-414.

Rutherford KM, Robson SK, Donald RD, Jarvis S, Sandercock DA, Scott EM, Nolan AM and Lawrence AB (2009). Pre-natal stress amplifies the immediate behavioural responses to acute pain in piglets. Biology Letters 5: 452-454.

Schmidt T, Konig A and Von Borell E (2012). Impact of general injection anaesthesia and analgesia on post-castration behaviour and teat order of piglets. Animal 6: 1998-2002.

Schonreiter S, Lohmuller V, Huber H, Zanella AJ, Unshelm J and Erhardt W (2000). Effects of $\mathrm{CO}_{2} / \mathrm{O}_{2}$-anaesthesia on behaviour, beta-endorphin- and cortisol concentrations of male piglets after castration. KTBL-Schrift 391: 137-145.

Schulz C, Ritzmann M, Palzer A, Heinritzi K and Zöls S. (2007a). Effect of isoflurane-anesthesia on postoperative pain due to castration of piglets. Berliner Munchener Tierarztliche Wochenschrift 120: 177-182.

Schulz C, Ritzmann M, Palzer A, Heinritzi K and Zoels S (2007b). Effect of isoflurane-anesthesia on postoperative pain due to castration of piglets. Berliner Munchener Tierarztliche Wochenschrift 120: 177-182.

Schwab S, Follrich B, Kurtev V and Keita A (2012). Ketoprofen practical use and efficacy for post-surgical analgesia in piglet castration Ketoprofen - Einsatz und Wirksamkeit zur post-operativen Analgesie bei der Ferkelkastration. Tierarztliche Umschau 67: 207-213.
Sutherland MA, Davis BL, Brooks TA and Mcglone JJ (2010). Physiology and behavior of pigs before and after castration: effects of two topical anesthetics. Animal 4: 2071-2079.

Sutherland MA, Davis BL and Mcglone JJ (2011). The effect of local or general anesthesia on the physiology and behavior of tail docked pigs. Animal 5: 1237-1246.

Sutherland MA, Davis BL, Brooks TA and Coetzee JF (2012). The physiological and behavioral response of pigs castrated with and without anesthesia or analgesia. Journal of Animal Science 90: 2211-2221.

Tenbergen R (2012). Investigation of the Use of Analgesics at the Time of Castration and Tail-docking and Following Parturition for Improving Performance and Reducing Pain in Pigs. Master of Science in Population Medicince, University of Guelph.

Waldmann KH, Otto K and Bollwahn W (1994). Castration of piglets: pain sensibility and anaesthesia. Deutsche Tierarztliche Wochenschrift 101: 105-109.

Walker B, Schatzmann U, Doherr M and Jaggin N (2004). Inhalation anaesthesia for castration of newborn piglets: experiences with isoflurane and isoflurane $\mathrm{N}_{2} \mathrm{O}$. Journal of Veterinary Medicine. Series A 51: 150-154.

Wavreille J, Danard M, Servais V, Art T, Nicks B and Laitat M (2012). Effect of preoperative meloxicam or tolfenamic acid administration on stress and pain induced by surgical castration in piglets. Journees de la Recherche Porcine en France 44: 275-276.

White RG, Deshazer JA, Tressler CJ, Borcher GM, Davey S, Waninge A, Parkhurst AM, Milanuk MJ and Clemens ET (1995). Vocalization and physiological response of pigs during castration with or without a local anesthetic. Journal of Animal Science 73: 381-386.

Zimmermann S, Zols S, Otten W, Palzer A, Ritzmann M and Heinritzi K (2011). Evaluation of carbon dioxide anaesthesia for the castration of male suckling piglets by stress hormone concentrations, behaviour and clinical factors. Berliner und Munchener Tierarztliche Wochenschrift 124: 368-375.

Zoels S, Ritzmann M and Heinritzi K (2006). Effect of analgesics on castration of male piglets. Berliner Munchener Tierarztliche Wochenschrift 119: 193-196. 